\title{
Vertical Discretization for a Nonhydrostatic Atmospheric Model Based on High-Order Spectral Elements
}

\author{
TAE-HYEONG YI \\ Korea Institute of Atmospheric Prediction Systems, Seoul, South Korea \\ FRANCIS X. GIRALDO \\ Department of Applied Mathematics, Naval Postgraduate School, Monterey, California
}

(Manuscript received 9 August 2018, in final form 11 October 2019)

\begin{abstract}
This study addresses the treatment of vertical discretization for a high-order, spectral element model of a nonhydrostatic atmosphere in which the governing equations of the model are separated into horizontal and vertical components by introducing a coordinate transformation, so that one can use different orders and types of approximations in both directions. The vertical terms of the decoupled governing equations are discretized using finite elements based on either Lagrange or basis-spline polynomial functions in the sigma coordinate, while maintaining the high-order spectral elements for the discretization of the horizontal terms. This leads to the fact that the high-order model of spectral elements with a nonuniform grid, interpolated within an element, can be easily accommodated with existing physical parameterizations. Idealized tests are performed to compare the accuracy and efficiency of the vertical discretization methods, in addition to the central finite differences, with those of the standard high-order spectral element approach. Our results show, through all the test cases, that the finite element with the cubic basis-spline function is more accurate than the other vertical discretization methods at moderate computational cost. Furthermore, grid dependency studies in the tests with and without orography indicate that the convergence rate of the vertical discretization methods is lower than the expected level of discretization accuracy, especially in the Schär mountain test, which yields approximately first-order convergence.
\end{abstract}

\section{Introduction}

Traditionally, hydrostatic models use pressure-based vertical coordinates (Thuburn 2011), where the vertical terms of the governing equations in terrain-following coordinates are typically discretized through central finite difference methods (FDM) (Taylor and Fournier 2010; ECMWF 2017). With computing power becoming increasingly accessible, many operational weather centers are moving toward solving fully nonhydrostatic equations, even for global weather prediction (Skamarock and Klemp 2008; Walters et al. 2011). For the vertical discretization, however, most global or limited-area nonhydrostatic models (Bonaventura 2000; Xue et al. 2000; Gassmann 2005; Satoh et al. 2014) are still based on the central FDM of Simmons and Burridge (1981), which requires the computational grid to be orthogonal

\footnotetext{
Corresponding author: Tae-Hyeong Yi, th.yi@kiaps.org
}

in order to separate the horizontal from the vertical directions. For this reason, a terrain-following coordinate transformation has been used in these models, whereby $x-z$ coordinates in a two-dimensional space are mapped onto sigma $(\sigma)$ (Phillips 1957; Gal-Chen and Somerville 1975 ), hybrid $\sigma$-pressure (Simmons and Burridge 1981; ECMWF 2017), or smooth level vertical (SLEVE) coordinates (Schär et al. 2002), in which the computational grid is then perfectly aligned and orthogonal with respect to each other. Among these terrain-following coordinates, the $\sigma$ coordinate is relatively simple and formulated with the pressure (Phillips 1957) or height (Gal-Chen and Somerville 1975) divided by its surface value. The hybrid $\sigma$-pressure coordinate, defined by the combination of $\sigma$ near the lower levels and the pressure at the upper levels was introduced by Simmons and Burridge (1981) to reduce numerical errors in the horizontal pressure gradient over steep orography (Sundqvist 1975; Haney 1991; Schär et al. 2002; Klemp et al. 2003), 
which is usually large in the $\sigma$ coordinate because of the appearance of steep orography profiles on stratospheric levels. This error is further reduced by using the SLEVE coordinate, which yields substantially smoother grids at the middle and upper levels, resulting in a reduction in transformation errors (Schär et al. 2002). The introduction of terrain-following coordinates, however, leads to severe misrepresentation in the surface properties near areas with steep orography due to smoothed terrain slopes (Thuburn 2011).

On the other hand, element-based Galerkin methods, such as continuous Galerkin (CG) finite element (Savre et al. 2016), spectral element (SE) (Giraldo and Rosmond 2004; Thomas and Loft 2005; Taylor and Fournier 2010; Kelly and Giraldo 2012) and discontinuous Galerkin (DG) finite element methods (Giraldo et al. 2002; Eskilsson and Sherwin 2004; Nair et al. 2005; Restelli and Giraldo 2009; Abdi and Giraldo 2016) have been widely adopted in atmospheric modeling because of their geometric flexibility, easy construction of highorder spatial discretization and, particularly, high scalability in massive parallel computing (Müller et al. 2018; Abdi et al. 2019). These standard finite elements do not require the coordinate transformation because the solution is constructed element-by-element. This allows the geometry of the element to be curvilinear in order to follow the terrain without requiring the elements to be perfectly aligned with the coordinate system. In such element-based Galerkin methods, the curvature of the element is handled through the metric terms that are naturally constructed by the Galerkin machinery typically through isoparametric mappings whereby the geometry is handled with the same order of accuracy as the numerical discretization which allows for consistency across all approximations. However, there may also exist additional reasons for requiring the use of such coordinates with the CG, SE, or DG method. One possible reason is due to the adoption of different numerical methods for the horizontal and vertical components. Without the introduction of terrain-following coordinates, one cannot decouple the horizontal from the vertical in the presence of orography. Another reason to decouple the horizontal and vertical directions would be to simplify the solution algorithm. Once decoupled, a tensor product of one-dimensional (1D) operators can be constructed separately for the horizontal and the vertical components, which may increase efficiency (Leveque 2002). The most important reason for decoupling the horizontal and vertical directions is to more easily accommodate existing physical parameterizations, since coupling with physics would not require mapping back and forth between the standard physics grid and the nonuniform interpolation points of the high-order element-based Galerkin methods.

In addition to the central FDM of Simmons and Burridge (1981) for the vertical discretization, a few alternative methods have been reported in the literature, such as finite elements with basis-spline (b-spline) functions (Staniforth and Daley 1977; Untch and Hortal 2004; Simarro and Hortal 2012; Yi and Park 2017; Vivoda et al. 2018) and the staggered nodal finite element method (SNFEM) (Guerra and Ullrich 2016). The latter method uses a combination of the DG and SE methods on a staggered grid to eliminate computational modes and the need for a reference state at high-order accuracy. Most studies in the former are based on the spectral method for the horizontal discretization, except the work of Yi and Park (2017), and a linear or cubic b-spline polynomial function is used as a basis function for the vertical discretization. By using the finite element with the b-spline basis function for the vertical discretization, referred to as the vertical finite element (VFE) method, the existing physical parameterizations developed for a Lorenz (Lorenz 1960) staggering grid system can be easily coupled to the highorder SE model without any modifications, since all dynamical and physical variables in the VFE method are defined at full levels (Yi and Park 2017). On the other hand, the variables with the central FDM of Simmons and Burridge (1981) are defined at either full or half levels. The VFE method has many advantages over the conventional central FDM, which is first-order accurate or second-order with careful treatment of metric terms in a nonuniform grid. In addition to enabling convenient coupling with the physical parameterizations in its framework, the VFE method presents highly accurate results with a cubic b-spline function and requires fewer grid points than the FDM at the same discretization accuracy (Untch and Hortal 2004; Vivoda et al. 2018). Although a third-order cubic b-spline function is implemented in the VFE method, its computational cost is not more expensive than a linear b-spline function (Yi and Park 2017). Moreover, the computational noise in the vertical direction can be reduced (Untch and Hortal 2004; Yi and Park 2017), compared with the central FDM of Simmons and Burridge (1981), where it inherently produces computational modes. As reported by $\mathrm{Yi}$ and Park (2017), the hydrostatic model with the VFE method provides better overall precipitation pattern and forecast skill errors in the short-range hindcasting case, compared with the central FDM of Simmons and Burridge (1981). For these reasons, we describe the construction of a $\sigma$ terrain-following coordinate to separate the governing equations into both directions 
for a standard high-order SE model. Once the horizontal and vertical terms are decoupled, we treat the vertical discretization with the VFE, vertical continuous Galerkin (VCG) and vertical finite difference (VFD) methods in which the second approach is finite elements based on Lagrange polynomial functions and the last is the secondorder central FDM (Pletcher et al. 2011).

This work is based on the two-dimensional (2D) nonhydrostatic equations in perturbation form with gravitational and Coriolis source terms, presented in section 2. The spatial discretization of the coupled governing equations is described along with the high-order SE method (SEM) in section 3. For time integration, an explicit, strong stability preserving, Runge-Kutta (SSP-RK) scheme is adopted in this study because of its high-order accuracy and simplicity. To decouple the governing equations into horizontal and vertical directions, the transformation of the equations in the $x-z$ coordinates into the $x-\sigma$ terrain-following coordinates is described in section 4 , where the derivation of the completely separated horizontal and vertical terms is provided. Moreover, a detailed description of the VFE method is discussed with a cubic b-spline function. Section 5 presents four idealized tests that are performed to compare the accuracy and efficiency of the decoupling approaches in terms of error norms and computational cost, which include the tests of rising thermal bubble, linear hydrostatic and nonhydrostatic waves over a small mountain, and Schär mountain waves. Some closing remarks are presented in section 6 .

\section{2D nonhydrostatic equations}

To simplify the discussion, the equations and its discretization are described in the context of an $x-z$ slice model. However, it should be understood that the same procedure is directly applicable to three dimensions. The nonconservative form of the nonhydrostatic equations in Cartesian coordinates can be written as

$$
\frac{\partial \mathbf{q}}{\partial t}+\mathbf{u} \cdot \nabla \mathbf{q}=S(\mathbf{q})
$$

where the components are given by

$$
\begin{aligned}
& \frac{\partial \rho}{\partial t}+\mathbf{u} \cdot \nabla \rho+\rho \nabla \cdot \mathbf{u}=0, \\
& \frac{\partial \mathbf{u}}{\partial t}+\mathbf{u} \cdot \nabla \mathbf{u}+\frac{1}{\rho} \nabla p=-g \hat{\mathbf{k}}-f \hat{\mathbf{k}} \times \mathbf{u},
\end{aligned}
$$

and

$$
\frac{\partial \theta}{\partial t}+\mathbf{u} \cdot \nabla \theta=0
$$

In the above equations, $S$ denotes the source term, defined as $(0,-g \hat{\mathbf{k}}-f \hat{\mathbf{k}} \times \mathbf{u}, 0)$, the prognostic variables are $(\rho, \mathbf{u}, \theta), \rho$ is the density, $\mathbf{u}=(u, w)$ is the Cartesian velocity field, $\theta$ is the potential temperature, $\nabla$ is the two-dimensional gradient operator, $\hat{\mathbf{k}}$ is the unit vector pointing in the $z$ direction, and $f$ is the Coriolis parameter. The pressure $p$ that appears in the momentum equation in Eq. (3) is obtained from the equation of state:

$$
p=p_{0}\left(\frac{\rho R \theta}{p_{0}}\right)^{\gamma}
$$

where the reference surface pressure is $p_{0}=10^{5} \mathrm{~Pa}$, the gas constant for dry air is $R=287 \mathrm{~J} \mathrm{~kg}^{-1} \mathrm{~K}^{-1}$, and the ratio of specific heats is $\gamma=1.4$.

By introducing the following splitting of the density $\rho(\mathbf{x}, t)=\bar{\rho}(z)+\rho^{\prime}(\mathbf{x}, t)$, where $\mathbf{x}=(x, z)$ and the reference variables, indicated by the superscript bar, are in hydrostatic balance (i.e., $d \bar{p} / d z=-g \bar{\rho}$ ), the potential temperature $\theta(\mathbf{x}, t)=\bar{\theta}(z)+\theta^{\prime}(\mathbf{x}, t)$, and the pressure $p(\mathbf{x}, t)=\bar{p}(z)+p^{\prime}(\mathbf{x}, t)$, Eqs. (2)-(4) can be rewritten in perturbation form to yield

$$
\begin{array}{r}
\frac{\partial \rho^{\prime}}{\partial t}+\mathbf{u} \cdot \nabla\left(\bar{\rho}+\rho^{\prime}\right)+\left(\bar{\rho}+\rho^{\prime}\right) \nabla \cdot \mathbf{u}=0 \\
\frac{\partial \mathbf{u}}{\partial t}+\mathbf{u} \cdot \nabla \mathbf{u}+\frac{1}{\bar{\rho}+\rho^{\prime}} \nabla p^{\prime}+\frac{\rho^{\prime}}{\bar{\rho}+\rho^{\prime}} g \hat{\mathbf{k}}+f \hat{\mathbf{k}} \times \mathbf{u}=0
\end{array}
$$

and

$$
\frac{\partial \theta^{\prime}}{\partial t}+\mathbf{u} \cdot \nabla\left(\bar{\theta}+\theta^{\prime}\right)=0
$$

where the superscript prime denotes the perturbation variables. For a general formulation that handles Cartesian and spherical geometries, the reader is referred to Giraldo et al. (2013).

\section{2D SE model}

\section{a. Basis functions and metric terms}

In the SEM, where Lagrange polynomials associated with the Legendre-Gauss-Lobatto (LGL) points are used as a basis function, a solution vector $\mathbf{q}$ is represented by the following $N$ th-degree basis function expansion, defined as

$$
\mathbf{q}_{N}(\mathbf{x}, t)=\sum_{i=1}^{M_{N}} \psi_{i}(\mathbf{x}) \mathbf{q}_{i}(t)
$$


On tensor-product grids (such as those used in this study), the basis functions $\psi_{i}$, given in Eq. (9), are constructed by a tensor product of one-dimensional basis functions such that $\psi_{i}(\xi, \eta)=h_{m}(\xi) \otimes h_{n}(\eta)$, where $\xi$ and $\eta$ are the coordinate components of a computational domain such as $\boldsymbol{\xi}=(\xi, \eta)$, and $\mathbf{q}_{i}$ represent the solutions at specific element points $i$. The subscript $N$ is used to denote the construction of an $N$ th-degree approximation to the state vector $\mathbf{q}$. The constant $M_{N}$ defines the number of basis functions required to build the $N$ th-degree approximation, where $M_{N}=(N+1)^{2}$ on quadrilateral elements. To employ the SEM in weak form, the integral of Eq. (9) for each element $\Omega_{e}$ is computed by Gaussian quadrature with the mapping from the physical domain $\mathbf{x}$ to the computational domain $\boldsymbol{\xi}$, confined by $\mathrm{I}=[-1,1]^{2}$, to yield

$$
\begin{aligned}
\int_{\Omega_{e}} \mathbf{q}_{N}(\mathbf{x}, t) d \mathbf{x} & =\int_{\mathbf{I}} \mathbf{q}_{N}(\mathbf{x}(\boldsymbol{\xi}), t)|J(\xi)| d \boldsymbol{\xi} \\
& \simeq \sum_{k=1}^{M_{Q}} \omega_{k} \mathbf{q}_{k}(t)\left|J\left(\xi_{k}, \eta_{k}\right)\right|,
\end{aligned}
$$

where $M_{Q}$ is the number of quadrature points of order $Q, \omega_{k}$ is the weight of the Gaussian quadrature, and $|J|$ is the determinant of the transformation Jacobian, where $J=\partial \mathbf{x} / \partial \boldsymbol{\xi}$. Local element-wise derivatives with respect to the space and time are constructed as

$$
\left.\frac{\partial \mathbf{q}_{N}(\mathbf{x}, t)}{\partial \mathbf{x}}\right|_{\Omega_{e}}=\sum_{i=1}^{M_{N}} \frac{d \psi_{i}(\mathbf{x})}{d \mathbf{x}} \mathbf{q}_{i}(t)
$$

and

$$
\left.\frac{\partial \mathbf{q}_{N}(\mathbf{x}, t)}{\partial t}\right|_{\Omega_{e}}=\sum_{i=1}^{M_{N}} \psi_{i}(\mathbf{x}) \frac{d \mathbf{q}_{i}(t)}{d t},
$$

respectively. For a description of possible basis functions, see Giraldo and Restelli (2008) for quadrilateral basis functions, Giraldo (2006) for triangular basis functions, and Kelly and Giraldo (2012) for hexahedral basis functions.

Since all the computations are performed in the computational domain with coordinates $\boldsymbol{\xi}$, the derivatives of the basis functions with respect to $\mathbf{x}$, given in Eq. (11), are transformed from $\mathbf{x}$ to $\boldsymbol{\xi}$, using the chain rule such that

$$
\frac{\partial \psi}{\partial \mathbf{x}}=\frac{\partial \psi}{\partial \xi} \frac{\partial \xi}{\partial \mathbf{x}}+\frac{\partial \psi}{\partial \eta} \frac{\partial \eta}{\partial \mathbf{x}}
$$

The metric terms $\partial \boldsymbol{\xi} / \partial \mathbf{x}$ are defined as

$$
\begin{aligned}
& \frac{\partial \xi}{\partial x}=\frac{1}{|J|} \frac{\partial z}{\partial \eta}, \quad \frac{\partial \xi}{\partial z}=-\frac{1}{|J|} \frac{\partial x}{\partial \eta}, \quad \frac{\partial \eta}{\partial x}=-\frac{1}{|J|} \frac{\partial z}{\partial \xi}, \quad \text { and } \\
& \frac{\partial \eta}{\partial z}=\frac{1}{|J|} \frac{\partial x}{\partial \xi}
\end{aligned}
$$

where the Jacobian determinant is given as

$$
|J|=\frac{\partial x}{\partial \xi} \frac{\partial z}{\partial \eta}-\frac{\partial z}{\partial \xi} \frac{\partial x}{\partial \eta}
$$

The derivatives of the physical coordinates $\partial \mathbf{x} / \partial \boldsymbol{\xi}$, given in Eq. (13), are approximated by the derivatives of the basis functions to yield

$$
\frac{\partial \mathbf{x}}{\partial \boldsymbol{\xi}}=\sum_{i=1}^{M_{N}} \frac{\partial \psi_{i}(\xi, \eta)}{\partial \boldsymbol{\xi}} \mathbf{x}_{i}
$$

where the derivative of the basis function with respect to $\boldsymbol{\xi}$ is easily computed with the Lagrangian polynomials used in the SEM.

\section{b. Spatial discretization with SEM}

To discretize the spatial terms of the governing equations, given in Eq. (1), with the weak form of the SEM, the equations are multiplied by basis function $\psi$ and integrated over each element, such that

$$
\int_{\Omega_{e}} \psi\left[\frac{\partial \mathbf{q}_{N}^{(e)}}{\partial t}+\mathbf{u}_{N}^{(e)} \cdot \nabla \mathbf{q}_{N}^{(e)}\right] d \Omega_{e}=\int_{\Omega_{e}} \psi S\left(\mathbf{q}_{N}^{(e)}\right) d \Omega_{e}
$$

where $\mathbf{q}_{N}^{(e)}$ denotes the degrees of freedom collocated in $\Omega_{e}$ and $\mathbf{u}_{N}^{(e)}$ is the velocity vector. ${ }^{1}$ Equation (17) may be written in a locally semidiscrete matrix form to yield

$$
\mathbf{M}^{(e)} \frac{d \mathbf{q}^{(e)}}{d t}+\mathbf{u}^{(e)} \mathbf{D}^{(e)} \mathbf{q}^{(e)}=\mathbf{S}\left(\mathbf{q}^{(e)}\right),
$$

where the local element-wise mass and differentiation matrices are defined on the reference element as

$$
\mathbf{M}_{i j}^{(e)}=\int_{\Omega_{e}} \psi_{i} \psi_{j} d \Omega_{e}
$$

and

$$
\mathbf{D}_{i j}^{(e)}=\int_{\Omega_{e}} \psi_{i} \nabla \psi_{j} d \Omega_{e}
$$

\footnotetext{
${ }^{1}$ To ensure mass conservation, we need to discretize the density equation differently, by not relying on a discrete product rule which, in general, will not hold with inexact integration, that is, we must discretize it in its original conservation form.
} 
respectively, where $i, j=1, \ldots, M_{N}$. To obtain the global solution with the SEM, the local element-wise matrices, given in Eqs. (19) and (20), are summed over all the elements, known as the direct stiffness summation:

$$
\mathbf{M}=\Lambda_{e=1}^{N_{e}} \mathbf{M}^{(e)}
$$

and

$$
\mathbf{D}=\Lambda_{e=1}^{N_{e}} \mathbf{D}^{(e)},
$$

where $\Lambda_{e=1}^{N_{e}}$ represents the mapping of the local-toglobal degrees of freedom for each element. This process ensures the continuity of the local solutions at the boundaries of each element in the computational domain. See Giraldo and Restelli (2008) for further details.

\section{c. Explicit Runge-Kutta time integration}

To advance the solution in time, the spatial and temporal terms of the governing equations given in Eq. (1) are rewritten with the method of lines, so that those terms are completely separated as

$$
\frac{d \mathbf{q}}{d t}=\mathbf{R}(\mathbf{q})
$$

where $\mathbf{q}$ is a prognostic variable vector and $\mathbf{R}$ is a residual that includes all the spatial, source and dissipative terms. Throughout this study, the third-order, five-stage, explicit SSP-RK (Spiteri and Ruuth 2002) is adopted for time integration, given as

$$
\begin{aligned}
\mathbf{q}^{0}= & \mathbf{q}^{n}, \\
\mathbf{q}^{k}= & \alpha_{0}^{k} \mathbf{q}^{n}+\alpha_{1}^{k} \mathbf{q}^{k-1}+\alpha_{2}^{k} \mathbf{q}^{k-3} \\
& +\beta^{k} \Delta t \mathbf{R}\left(\mathbf{q}^{k-1}\right), \quad \text { where } \quad k=1, \ldots, s, \quad \text { and } \\
\mathbf{q}^{n+1}= & \mathbf{q}^{s},
\end{aligned}
$$

where the superscripts $n$ and $n+1$ indicate the present and subsequent time levels, respectively, $s$ denotes the number of stages, and the coefficients $\alpha$ and $\beta$ are given in Spiteri and Ruuth (2002). In the equations, $\mathbf{q}^{k-3}$ is valid only in the last stage. Giraldo and Restelli (2010) reported that this RK scheme has a larger stability region than the third-order, three-stage explicit RK of Shu and Osher (1988). Although the explicit RK scheme considerably limits the time step in the nonhydrostatic equations, compared with fully implicit or semi-implicit scheme, it is easier to implement and is sufficient for the purposes of this study. It should be noted that the general solution strategy described in this study can be applied directly to semi-implicit time integration methods.
For the explicit RK scheme, the Courant numbers in the horizontal and vertical directions are respectively defined as (Blazek 2015)

$$
C_{h}=\frac{\Delta t(u+a)}{\Delta h} \quad \text { and } \quad C_{v}=\frac{\Delta t(w+a)}{\Delta v},
$$

where $\Delta t$ is a time step, $a$ is the speed of sound, and $\Delta h$ and $\Delta v$ are the horizontal and vertical spacing, respectively, between the LGL points or uniform grids. The time step is chosen such that $C_{h}$ and $C_{v}$ are less than unity to maintain the stability of the model.

\section{d. Boundary conditions}

In this study, no-flux and nonreflecting boundary conditions are utilized, depending on the problem. No-flux boundary conditions are always used along the bottom boundary while either no-flux or nonreflecting boundary conditions are used along the lateral and top boundaries, depending on the test case. The equation and description of those boundary conditions for the CG finite elements can be found in Kelly and Giraldo (2012). However, for completeness, we now define the sponge nonreflecting boundary condition. The Newtonian relaxation coefficient near the top, $\beta(z)$, is constructed as follows:

$$
\beta(z)=\left\{\begin{array}{cl}
0, & \text { if } z<z_{T}-z_{b} \\
\tau_{\text {top }}\left(\frac{z-z_{b}}{z_{T}-z_{b}}\right)^{4}, & \text { otherwise }
\end{array},\right.
$$

where $z_{T}$ is the domain height, $z_{b}$ is the bottom location of the sponge layer, and $\tau_{\text {top }}$ is a sponge coefficient. The Newtonian relaxation coefficient near the lateral boundary, $\beta(x)$, is obtained by a similar manner. The solution vector is then updated by

$$
\mathbf{q}=\alpha(\mathbf{x}) \mathbf{q}_{R}+\beta(\mathbf{x}) \mathbf{q}_{b},
$$

where $\mathbf{q}_{R}$ is the solution obtained from each timeintegration step, $\mathbf{q}_{b}$ is the given value at the boundary, and the Newtonian relaxation coefficients $\beta(\mathbf{x})$ and $\alpha(\mathbf{x})$ are defined as $\beta(\mathbf{x})=\beta(x)+\beta(z)$ and $\alpha(\mathbf{x})=1-\beta(\mathbf{x})$, respectively. This defines a simple nonreflecting boundary condition but there are numerous other more sophisticated choices. The interested reader is referred to Appelö and Kreiss (2006), Dea et al. (2009) and Lindquist et al. (2012).

\section{Vertical discretization in terrain-following coordinates}

The SE machinery described in section 3 allows one to solve the governing equations directly, without concern that the grid boxes are warped (nonorthogonal), in 
spite of the fact that gravity acts downward (in the direction of the negative $z$ axis) and thereby possibly across vertical grid lines. The benefit of using the SEM is that the above factors are accounted for as long as the grid is valid to solve the equations [i.e., the Jacobian of the transformation from $(x, z)$ to $(\xi, \eta)$ is greater than zero]. Thus, it is natural to solve a horizontally and vertically coupled problem, but there may be reasons to separate the horizontal and vertical discretization. One example is to allow the use of a more uniform grid spacing in the vertical direction than the LGL grid to couple with the physics. In the SEM, where a basis function and a quadrature rule are defined on the same grid points, leading to a diagonal mass matrix, the spatial discretization error is almost negligible for sufficiently large $N(\geq 4)$, but for $N<4$, the error becomes quite large (Giraldo 1998). Therefore, if one wishes to use $N<4$, it then requires using different integration points (i.e., the $\mathrm{CG}$ method with exact integration using Gauss quadrature, such as $N+1$ LGL or $N$ Legendre-Gauss points), which causes the mass matrix to no longer be diagonal but rather sparse and global. This requires the inversion of a global and sparse mass matrix at each time iteration, resulting in significantly more computational cost. However, if the governing equations are separated into the horizontal and vertical directions and the vertical grid is uniform $\left(N_{z}=1\right.$ or 2$)$, the vertical mass matrix can be quite small and thus, feasible for inversion. Based on this uniform grid, the vertical terms of the governing equations decoupled in the $x-\sigma$ terrain-following coordinates are discretized with the CG finite element, referred to as VCG, and VFE methods. Figure 1 shows a typical example of such a coordinate transformation with horizontal LGL and vertically uniform grids.

\section{a. Transformation to terrain-following coordinates}

The nonhydrostatic equations given by Eqs. (6)-(8) are written in terms of the Cartesian $x-z$ coordinates. However, the grid boxes are not necessarily aligned with the $x-z$ coordinates, which makes it impossible to decouple the horizontal and vertical directions. To decouple the directions, a coordinate system from $(x, z)$ to $(\tilde{x}, \tilde{z})$ is introduced. The classical way to derive these coordinates is via the chain rule as follows:

$$
\left(\begin{array}{l}
d \tilde{x} \\
d \tilde{z}
\end{array}\right)=\left(\begin{array}{ll}
\frac{\partial \tilde{x}}{\partial x} & \frac{\partial \tilde{x}}{\partial z} \\
\frac{\partial \tilde{z}}{\partial x} & \frac{\partial \tilde{z}}{\partial z}
\end{array}\right)\left(\begin{array}{l}
d x \\
d z
\end{array}\right)=\tilde{\mathbf{J}}\left(\begin{array}{l}
d x \\
d z
\end{array}\right),
$$

where $\tilde{\mathbf{J}}$ denotes the Jacobian of the coordinate transformation. Gal-Chen and Somerville (1975) proposed
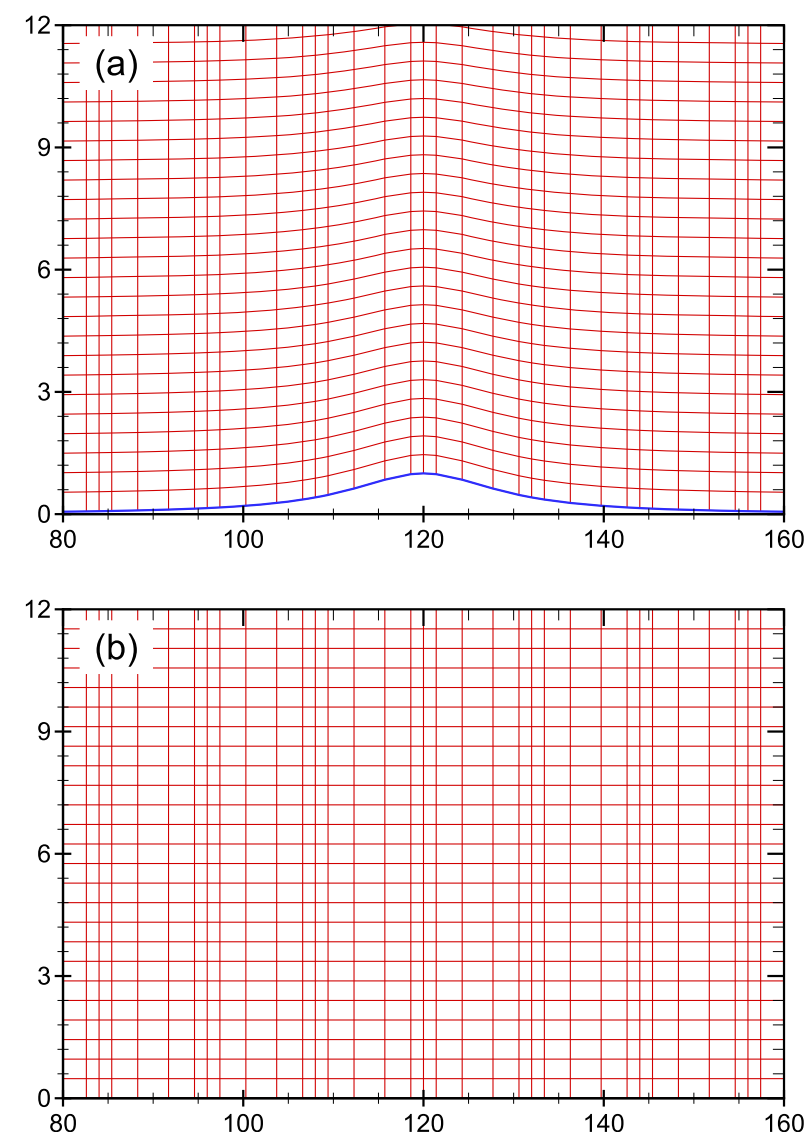

FIG. 1. Transformation of (a) $x-z$ to (b) $x-\sigma$ coordinates with horizontal LGL and vertically uniform grids used for the test of mountain waves, where the $1-\mathrm{km}$-high mountain is used. Spatial units are in kilometers.

the following terrain-following coordinate transformation, defined as

$$
\tilde{x}=x
$$

and

$$
\tilde{z}=z_{T}\left(\frac{z-z_{S}}{z_{T}-z_{S}}\right)
$$

where $z_{S}=z_{S}(x)$ is the representation of the surface height with respect to the $x$ coordinate. In fact, $\tilde{z}=\sigma$ and is typically referred to as $\sigma-z$ coordinates. From Eq. (28), replacing $d \mathbf{x}$ with $\mathbf{u}=d \mathbf{x} / d t$ allows one to transform the velocity and gradient operator into $\tilde{\mathbf{u}}=\tilde{\mathbf{J}} \mathbf{u}$ and $\boldsymbol{\nabla}=\tilde{\mathbf{J}} \widetilde{\nabla}$, respectively, where $\mathscr{T}$ is the transpose operator. With the Gal-Chen and Somerville transformation, the Jacobian given in Eq. (28) becomes

$$
\tilde{\mathbf{J}}=\left(\begin{array}{cc}
1 & 0 \\
\frac{\partial \sigma}{\partial x} & \frac{\partial \sigma}{\partial z}
\end{array}\right),
$$


where

$$
\frac{\partial \sigma}{\partial x}=\frac{\sigma-z_{T}}{z_{T}-z_{S}} \frac{d z_{S}}{d x}
$$

and

$$
\frac{\partial \sigma}{\partial z}=\frac{z_{T}}{z_{T}-z_{S}} .
$$

Substituting the above transformation for the velocity and gradient operators into Eqs. (6)-(8) yields the following equations:

$$
\frac{\partial \rho^{\prime}}{\partial t}+(\tilde{\mathbf{u}} \cdot \tilde{\nabla})\left(\bar{\rho}+\rho^{\prime}\right)+\left(\bar{\rho}+\rho^{\prime}\right) \tilde{\nabla} \cdot \tilde{\mathbf{u}}=0,
$$

$$
\frac{\partial \mathbf{u}}{\partial t}+(\tilde{\mathbf{u}} \cdot \tilde{\mathbf{\nabla}}) \mathbf{u}+\frac{1}{\bar{\rho}+\rho^{\prime}} \tilde{\mathbf{J}} \cdot \tilde{\nabla} p^{\prime}+\frac{\rho^{\prime}}{\bar{\rho}+\rho^{\prime}} g \hat{\mathbf{k}}+f \hat{\mathbf{k}} \times \mathbf{u}=0,
$$

and

$$
\frac{\partial \theta^{\prime}}{\partial t}+(\tilde{\mathbf{u}} \cdot \tilde{\mathbf{\nabla}})\left(\bar{\theta}+\theta^{\prime}\right)=0
$$

For the details of the transformation and the resulting equations, the reader is referred to Gibbons (2009).

To understand the discretization of Eqs. (34)(36) by the decoupled SEM, the equations are expanded into each separate component. Recalling that $(\tilde{x}, \tilde{z})=(x, \sigma)$ and $(\tilde{u}, \tilde{w})=(u, \dot{\sigma})$ allows us to recast Eqs. (34)-(36) as

$$
\begin{gathered}
\frac{\partial \rho^{\prime}}{\partial t}+u \frac{\partial \rho^{\prime}}{\partial x}+\dot{\sigma} \frac{\partial\left(\bar{\rho}+\rho^{\prime}\right)}{\partial \sigma}+\left(\bar{\rho}+\rho^{\prime}\right)\left(\frac{\partial u}{\partial x}+\frac{\partial \dot{\sigma}}{\partial \sigma}\right)=0 \\
\frac{\partial u}{\partial t}+u \frac{\partial u}{\partial x}+\dot{\sigma} \frac{\partial u}{\partial \sigma}+\frac{1}{\bar{\rho}+\rho^{\prime}}\left(\frac{\partial p^{\prime}}{\partial x}+\frac{\partial \sigma}{\partial x} \frac{\partial p^{\prime}}{\partial \sigma}\right)+f w=0 \\
\frac{\partial w}{\partial t}+u \frac{\partial w}{\partial x}+\dot{\sigma} \frac{\partial w}{\partial \sigma}+\frac{1}{\bar{\rho}+\rho^{\prime}} \frac{\partial \sigma}{\partial z} \frac{\partial p^{\prime}}{\partial \sigma}+\frac{\rho^{\prime}}{\bar{\rho}+\rho^{\prime}} g-f u=0
\end{gathered}
$$

and

$$
\frac{\partial \theta^{\prime}}{\partial t}+u \frac{\partial \theta^{\prime}}{\partial x}+\dot{\sigma} \frac{\partial\left(\bar{\theta}+\theta^{\prime}\right)}{\partial \sigma}=0
$$

In Eqs. (37)-(40), all the spatial derivatives are distinctly separated into horizontal and vertical operators in terms of the $x-\sigma$ coordinates. In the following sections, the discretization of the vertical operators with the VCG and VFE methods is discussed, while the horizontal operators are still treated with the high-order SE machinery.

\section{b. Vertical discretization with $C G$ method}

With the decoupled governing equations in the $x-\sigma$ terrain-following coordinates, the vertical operator is discretized with the CG finite element method. Let us rewrite Eq. (9) as a tensor product of 1D basis functions:

$$
\mathbf{q}_{N}(x, \sigma, t)=\sum_{i, j=0}^{N} h_{i}(\xi(x)) h_{j}(\eta(\sigma)) \mathbf{q}_{i j}(t),
$$

where $h$ is a $1 \mathrm{D}$ basis function. In this equation, $h_{i}$ or $h_{j}$ is only a function of $\xi=\xi(x)$ or $\eta=\eta(\sigma)$, respectively, because the terrain-following coordinates ensure that the gridlines with respect to $x$ and $\sigma$ are orthogonal. From Eq. (41), the derivatives with respect to $x$ and $\sigma$ are approximated, respectively, as

$$
\frac{\partial \mathbf{q}_{N}(x, \sigma, t)}{\partial x}=\sum_{i, j=0}^{N} \frac{d h_{i}(\xi(x))}{d \xi} \frac{d \xi}{d x} h_{j}(\eta(\sigma)) \mathbf{q}_{i j}(t)
$$

and

$$
\frac{\partial \mathbf{q}_{N}(x, \sigma, t)}{\partial \sigma}=\sum_{i, j=0}^{N} h_{i}(\xi(x)) \frac{d h_{j}(\eta(\sigma))}{d \eta} \frac{d \eta}{d \sigma} \mathbf{q}_{i j}(t),
$$

where $d \xi / d x=2 / \Delta x^{(e)}$ and $d \eta / d \sigma=2 / \Delta \sigma^{(e)}$ with $\Delta x^{(e)}$ and $\Delta \sigma^{(e)}$ as the length of the element in the $x(\xi)$ and $\sigma(\eta)$ directions, respectively.

With Eqs. (42) and (43), the decoupled discretization is applied to the continuity equation given in Eq. (37). This equation is rewritten with $\rho=\bar{\rho}+\rho^{\prime}$ as

$$
\frac{\partial \rho^{\prime}}{\partial t}+u \frac{\partial \rho^{\prime}}{\partial x}+\dot{\sigma} \frac{\partial \rho}{\partial \sigma}+\rho\left(\frac{\partial u}{\partial x}+\frac{\partial \dot{\sigma}}{\partial \sigma}\right)=0 .
$$

Multiplying Eq. (44) by $h_{i}(x) h_{j}(\sigma)$ and integrating yields the integral equation separated by the horizontal and vertical terms:

$$
\begin{aligned}
& \int_{x} h_{i}(x) h_{k}(x) d x \int_{\sigma} h_{j}(\sigma) h_{l}(\sigma) d \sigma \frac{d \rho_{k, l}^{\prime}}{d t}+ \\
& \int_{x} h_{i}(x) \frac{\partial h_{k}(x)}{\partial x} h_{m}(x) d x \int_{\sigma} h_{j}(\sigma) h_{l}(\sigma) h_{n}(\sigma) d \sigma \rho_{k, l}^{\prime} u_{m, n}+ \\
& \int_{x} h_{i}(x) h_{k}(x) h_{m}(x) d x \int_{\sigma} h_{j}(\sigma) \frac{\partial h_{l}(\sigma)}{\partial \sigma} h_{n}(\sigma) d \sigma \rho_{k, l} \dot{\sigma}_{m, n}+ \\
& \int_{x} h_{i}(x) \frac{\partial h_{k}(x)}{\partial x} h_{m}(x) d x \int_{\sigma} h_{j}(\sigma) h_{l}(\sigma) h_{n}(\sigma) d \sigma u_{k, l} \rho_{m, n}+ \\
& \int_{x} h_{i}(x) h_{k}(x) h_{m}(x) d x \int_{\sigma} h_{j}(\sigma) \frac{\partial h_{l}(\sigma)}{\partial \sigma} h_{n}(\sigma) d \sigma \dot{\sigma}_{k, l} \rho_{m, n}=0,
\end{aligned}
$$


where the tensor product of any scalar variable is approximated by Eq. (41). To better understand the indices in Eq. (45), it is important to note that we seek a solution to $\mathbf{q}_{N}$ in terms of the matrix indices $(i, j)$ that represent the interpolation points along the $x$ and $\sigma$ directions. Therefore, anytime an index is not in the first location of either an integral or an element matrix, it represents a summation, which means that index will vanish upon performing the sum. Next, transforming Eq. (45) into the computational coordinates $(\xi, \eta)$ gives

$$
\begin{aligned}
& \frac{\Delta x^{(e)}}{2} \frac{\Delta \sigma^{(e)}}{2} \int_{-1}^{+1} h_{i}(\xi) h_{k}(\xi) d \xi \int_{-1}^{+1} h_{j}(\eta) h_{l}(\eta) d \eta \frac{d \rho_{k, l}^{\prime}}{d t}+ \\
& \frac{\Delta \sigma^{(e)}}{2} \int_{-1}^{+1} h_{i}(\xi) \frac{d h_{k}(\xi)}{d \xi} h_{m}(\xi) d \xi \int_{-1}^{+1} h_{j}(\eta) h_{l}(\eta) h_{n}(\eta) d \eta \rho_{k, l}^{\prime} u_{m, n}+ \\
& \frac{\Delta x^{(e)}}{2} \int_{-1}^{+1} h_{i}(\xi) h_{k}(\xi) h_{m}(\xi) d \xi \int_{-1}^{+1} h_{j}(\eta) \frac{d h_{l}(\eta)}{d \eta} h_{n}(\eta) d \eta \rho_{k, l} \dot{\sigma}_{m, n}+ \\
& \frac{\Delta \sigma^{(e)}}{2} \int_{-1}^{+1} h_{i}(\xi) \frac{d h_{k}(\xi)}{d \xi} h_{m}(\xi) d \xi \int_{-1}^{+1} h_{j}(\eta) h_{l}(\eta) h_{n}(\eta) d \eta u_{k, l} \rho_{m, n}+ \\
& \frac{\Delta x^{(e)}}{2} \int_{-1}^{+1} h_{i}(\xi) h_{k}(\xi) h_{m}(\xi) d \xi \int_{-1}^{+1} h_{j}(\eta) \frac{d h_{l}(\eta)}{d \eta} h_{n}(\eta) d \eta \dot{\sigma}_{k, l} \rho_{m, n}=0 .
\end{aligned}
$$

If inexact and exact integrations are used for the $\xi$ and $\eta$ integrals, respectively, Eq. (46) is discretized using Eq. (10) to arrive at the following matrix equation:

$$
\mathbf{M}_{i}^{(\xi, e)} \mathbf{M}_{j, l}^{(\eta, e)} \frac{d \rho_{i, l}^{\prime}}{d t}+\mathbf{D}_{i, k}^{(\xi, e)} \mathbf{M}_{j, l, n}^{(\eta, e)} \rho_{k, l}^{\prime} u_{i, n}+\mathbf{M}_{i}^{(\xi, e)} \mathbf{D}_{j, l, n}^{(\eta, e)} \rho_{i, l} \dot{\sigma}_{i, n}+\mathbf{D}_{i, k}^{(\xi, e)} \mathbf{M}_{j, l, n}^{(\eta, e)} u_{k, l} \rho_{i, n}+\mathbf{M}_{i}^{(\xi, e)} \mathbf{D}_{j, l, n}^{(\eta, e)} \dot{\sigma}_{i, l} \rho_{i, n}=0
$$

where the matrices appearing in Eq. (47) are defined as

$$
\begin{aligned}
\mathbf{M}_{i}^{(\xi, e)} & =w_{i} \frac{\Delta x^{(e)}}{2}, \\
\mathbf{D}_{i, k}^{(\xi, e)} & =w_{i} \frac{d h_{k}\left(\xi_{i}\right)}{d \xi} \\
\mathbf{M}_{j, l}^{(\eta, e)} & =\frac{\Delta \sigma^{(e)}}{2} \sum_{q=0}^{N_{q}} w_{q} h_{j}\left(\eta_{q}\right) h_{l}\left(\eta_{q}\right), \\
\mathbf{M}_{j, l, n}^{(\eta, e)} & =\frac{\Delta \sigma^{(e)}}{2} \sum_{q=0}^{N_{q}} w_{q} h_{j}\left(\eta_{q}\right) h_{l}\left(\eta_{q}\right) h_{n}\left(\eta_{q}\right),
\end{aligned}
$$

and

$$
\mathbf{D}_{j, l, n}^{(\eta, e)}=\sum_{q=0}^{N_{q}} w_{q} h_{j}\left(\eta_{q}\right) \frac{d h_{l}\left(\eta_{q}\right)}{d \eta} h_{n}\left(\eta_{q}\right) .
$$

Here, $N_{q}$ is the number of quadrature points required to integrate $\mathbf{M}^{(\eta)}$ accurately. Using LGL points for interpolation and integration requires $Q_{2 N}=N+1$ and $Q_{3 N}=N+2$ integration points to achieve exact integration, where the subscript $d$ in $Q_{d}$ denotes the degree of the polynomial order. Therefore, we can choose to use these points to incur no integration error but at the cost of requiring the inversion of a global mass matrix.

Using the approximation ${ }^{2} \mathbf{M}_{j, l, n}^{(\eta, e)} \approx \mathbf{M}_{j, l}^{(\eta, e)}$ and multiplying Eq. (47) by the inverse of $\mathbf{M}_{i}^{(\xi, e)}$ and $\mathbf{M}_{j, l}^{(\eta, e)}$ yields the following simplification of Eq. (47):

$$
\begin{aligned}
& \frac{d \rho_{i, j}^{\prime}}{d t}+\hat{\mathbf{D}}_{i, k}^{(\xi, e)} \rho_{k, j}^{\prime} u_{i, j}+\hat{\mathbf{D}}_{j, l, n}^{(\eta, e)} \rho_{i, l} \dot{\sigma}_{i, n}+\hat{\mathbf{D}}_{i, k}^{(\xi, e)} u_{k, j} \rho_{i, j} \\
& \quad+\hat{\mathbf{D}}_{j, l, n}^{(\eta, e)} \dot{\sigma}_{i, l} \rho_{i, n}=0
\end{aligned}
$$

where

$$
\hat{\mathbf{D}}_{i, k}^{(\xi, e)}=\left[\mathbf{M}_{i}^{(\xi, e)}\right]^{-1} \mathbf{D}_{i, k}^{(\xi, e)}
$$

and

\footnotetext{
${ }^{2}$ We make the approximation $\mathbf{M}_{j, l, n}^{(\eta, e)} \approx \mathbf{M}_{j, l}^{(\eta, e)}$ to simplify the description of the method. However, this approximation is strictly not necessary. Making this approximation is equivalent to representing the quadratic term $\rho_{l} u_{n}$ grouped into the linear term $(\rho u)_{l}$ along the vertical direction only. This is typically done in, for example, the group finite element method and also to prove discrete integration by parts with Galerkin methods. These advantages outweigh the accuracy loss that may be incurred.
} 


$$
\hat{\mathbf{D}}_{j, l, n}^{(\eta, e)}=\left[\mathbf{M}_{j, l}^{(\eta, e)}\right]^{-1} \mathbf{D}_{j, l, n}^{(\eta, e)} .
$$

Equation (53) is the continuity equation, written in a form whereby the operators along $\xi$ and $\eta$ have been entirely decoupled. Similarly, the remaining equations given in Eqs. (38)-(40) are discretized with the horizontal and vertical derivative operators, respectively defined as

$$
\frac{\partial}{\partial x}=\hat{\mathbf{D}}_{i, k}^{(\xi, e)}
$$

and

$$
\frac{\partial}{\partial \sigma}=\hat{\mathbf{D}}_{j, l, n, d}^{(\eta, e)}
$$

where the last subscript $d$ in $\hat{\mathbf{D}}^{(\eta, e)}$ is either $r$ or has no value, depending on the dimensionality of the operator. In the continuity equation, for example, this operator has no value, but in the pressure gradient of the momentum equation, it has a value of $r$.

Let us now apply the approximations derived in this section to the nonhydrostatic equations. Using Eqs. (56) and (57), the semidiscrete forms of Eqs. (37)-(40) are rewritten as

$$
\begin{array}{r}
\frac{d \rho_{i, j}^{\prime}}{d t}+u_{i, j} \hat{\mathbf{D}}_{i, k}^{(\xi, e)} \rho_{k, j}^{\prime}+\rho_{i, j} \hat{\mathbf{D}}_{i, k}^{(\xi, e)} u_{k, j}+\hat{\mathbf{D}}_{j, l, n}^{(\eta, e)} \rho_{i, l} \dot{\sigma}_{i, n}+\hat{\mathbf{D}}_{j, l, n}^{(\eta, e)} \dot{\sigma}_{i, l} \rho_{i, n}=0 \\
\frac{d u_{i, j}}{d t}+u_{i, j} \hat{\mathbf{D}}_{i, k}^{(\xi, e)} u_{k, j}+\frac{1}{\rho_{i, j}} \hat{\mathbf{D}}_{i, k}^{(\xi, e)} p_{k, j}^{\prime}+\hat{\mathbf{D}}_{j, l, n}^{(\eta, e)} u_{i, l} \dot{\sigma}_{i, n}+\hat{\mathbf{D}}_{j, l, n, r}^{(\eta, e)} p_{i, l}^{\prime} \frac{1}{\rho_{i, n}} \frac{\partial \sigma_{i, p}}{\partial x}+f w_{i, j}=0 \\
\frac{d w_{i, j}}{d t}+u_{i, j} \hat{\mathbf{D}}_{i, k}^{(\xi, e)} w_{k, j}+\hat{\mathbf{D}}_{j, l, n}^{(\eta, e)} w_{i, l} \dot{\sigma}_{i, n}+\hat{\mathbf{D}}_{j, l, n, r}^{(\eta, e)} p_{i, l}^{\prime} \frac{1}{\rho_{i, n}} \frac{\partial \sigma_{i, p}}{\partial z}+\left(\frac{\rho^{\prime}}{\rho}\right)_{i, j} g-f u_{i, j}=0,
\end{array}
$$

and

$$
\frac{d \theta_{i, j}^{\prime}}{d t}+u_{i, j} \hat{\mathbf{D}}_{i, k}^{(\xi, e)} \theta_{k, j}^{\prime}+\hat{\mathbf{D}}_{j, l, n}^{(\eta, e)} \theta_{i, l}^{\prime} \dot{\sigma}_{i, n}+\hat{\mathbf{D}}_{j, l, n}^{(\eta, e)} \bar{\theta}_{i, l} \dot{\sigma}_{i, n}=0 .
$$

Equations (58)-(61) are the horizontally and vertically decoupled nonhydrostatic equations discretized with the SE and VCG methods, respectively, in the $x-\sigma$ terrainfollowing coordinates.

\section{c. VFE with b-spline basis function}

In this section, the discretization of the vertical terms given in Eqs. (37)-(40) is presented, using the Galerkin finite element method (FEM), but instead of using Lagrange polynomials based on the LGL grid points, $b$-spline polynomials are used as the basis functions on a uniform grid, while the horizontal terms are still approximated by the high-order SEM. As shown in Eqs. (37)-(40), the vertical components are derivatives with respect to $\sigma$, so that the terms to be discretized with the VFE method are derivative operators. In the work of Yi and Park (2017), the performance of the VFE with a linear b-spline function provided similar behavior to that of the VFD method, in terms of accuracy and stability, and for this reason, we mainly use a cubic b-spline basis function due to its high-order accuracy.

\section{1) B-SPLINE FUNCTION}

The VFE scheme employs the b-spline basis function, which forms piecewise polynomials using either a uniform or nonuniform grid. Similar to Eq. (9), the solution vector $\mathbf{q}$ is approximated by a linear combination of the b-spline basis functions $b(\sigma)$ and coefficients $\hat{\mathbf{q}}(t)$ :

$$
\mathbf{q}_{N}(\sigma, t)=\sum_{i=1}^{N_{p}} b_{i}(\sigma) \hat{\mathbf{q}}_{i}(t),
$$

where $N_{p}$ is the number of grid points. The set of the cubic b-spline functions $b_{i}(\sigma)$ is defined over four intervals on a uniform grid in analytic form (Prenter 1975; Untch and Hortal 2004):

$$
b_{i}(\sigma)=\frac{1}{4 h^{3}}\left\{\begin{array}{cll}
\left(\sigma-\sigma_{i-2}\right)^{3}, & \text { if } & \sigma \in\left[\sigma_{i-2}, \sigma_{i-1}\right] \\
h^{3}+3 h^{2}\left(\sigma-\sigma_{i-1}\right)+3 h\left(\sigma-\sigma_{i-1}\right)^{2}-3\left(\sigma-\sigma_{i-1}\right)^{3}, & \text { if } & \sigma \in\left[\sigma_{i-1}, \sigma_{i}\right] \\
h^{3}+3 h^{2}\left(\sigma_{i+1}-\sigma\right)+3 h\left(\sigma_{i+1}-\sigma\right)^{2}-3\left(\sigma_{i+1}-\sigma\right)^{3}, & \text { if } & \sigma \in\left[\sigma_{i}, \sigma_{i+1}\right] \\
\left(\sigma_{i+2}-\sigma\right)^{3}, & \text { if } & \sigma \in\left[\sigma_{i+1}, \sigma_{i+2}\right] \\
0, & \text { otherwise, }
\end{array}\right.
$$


where $h$ is the interval between levels. In Eq. (63), two additional knots are required to define the cubic b-spline polynomials on each lowermost and uppermost level, which are $\sigma_{-1}, \sigma_{0}$ and $\sigma_{N_{p}+1}, \sigma_{N_{p}+2}$, respectively, so that the number of cubic b-spline polynomials becomes $N_{p}+4$. Based on the choice of b-spline function, the components of the projection and mass matrices are determined, in which the projection and mass matrices for the cubic b-spline are tridiagonal and pentadiagonal, respectively. For a detailed description regarding the b-spline functions in analytic form, including the cubic b-spline function on a nonuniform grid, the reader is referred to Untch and Hortal (2004).

\section{2) VERTICAL DisCRETIZATION WITH VFE}

To solve differential equations, the discretization with the VFE method based on the b-spline basis function is expressed as a matrix representing the derivative operator. Let us consider the differential equation in the vertical direction, defined as

$$
F(\sigma)=\frac{d}{d \sigma} f(\sigma) .
$$

Approximating the physical functions $F(\sigma)$ and $f(\sigma)$ by the basis expansions given in Eq. (62), multiplying by the test function $b_{k}(\sigma)$ and integrating from the bottom to the top of the vertical domain yields the following equation expressed in the representation of the FEM:

$$
\sum_{i=1}^{N_{p}} \hat{F}_{i} \int_{\mathrm{btm}}^{\mathrm{top}} b_{i}(\sigma) b_{k}(\sigma) d \sigma=\sum_{j=1}^{N_{p}} \hat{f}_{j} \int_{\mathrm{btm}}^{\mathrm{top}} \frac{d b_{j}(\sigma)}{d \sigma} b_{k}(\sigma) d \sigma,
$$

where $\hat{F}_{i}$ and $\hat{f}_{j}$ denote the coefficients in the finite element space associated with the functions $F(\sigma)$ and $f(\sigma)$, respectively. To express the finite element representation of the vertical derivative operator in matrix form, the mass matrix $\mathbf{M}$ and stiffness matrix $\mathbf{S}$ are respectively defined as

$$
\mathbf{M}_{i k}=\int_{\mathrm{btm}}^{\mathrm{top}} b_{i}(\sigma) b_{k}(\sigma) d \sigma
$$

and

$$
\mathbf{S}_{j k}=\int_{\mathrm{btm}}^{\mathrm{top}} \frac{d b_{j}(\sigma)}{d \sigma} b_{k}(\sigma) d \sigma .
$$

Now we can recast Eq. (65) in matrix form of algebraic equations to yield

$$
\sum_{i=1}^{N_{p}} \hat{F}_{i} \mathbf{M}_{i k}=\sum_{j=1}^{N_{p}} \hat{f} \mathbf{S}_{j k} \quad \text { or } \quad \mathbf{M} \hat{\mathbf{F}}=\mathbf{S} \hat{\mathbf{f}} .
$$

Introducing the projection matrices $\mathbf{P}$ and $\mathbf{Q}$, the corresponding functions $F(\sigma)$ and $f(\sigma)$ are transformed from the finite element space to physical grid levels, which are, respectively, defined as (Untch and Hortal 2004; Simarro and Hortal 2012)

$$
F(\sigma)=\sum_{i=1}^{N_{p}} \hat{F}_{i} b_{i}(\sigma)=\mathbf{P} \hat{\mathbf{F}}
$$

and

$$
f(\sigma)=\sum_{j=1}^{N_{p}} \hat{f}_{j} b_{j}(\sigma)=\mathbf{Q} \hat{\mathbf{f}} .
$$

After multiplying both sides of Eq. (68) by $\mathbf{M}^{-1}$ and substituting into Eq. (69), the derivative function of Eq. (64) with respect to $\sigma$ becomes

$$
F(\sigma)=\frac{d}{d \sigma} f(\sigma)=\mathbf{P M}^{-1} \mathbf{S} \mathbf{Q}^{-1} f(\sigma)=\mathcal{D} f(\sigma),
$$

where $\mathcal{D}$ denotes the vertical derivative operator. Note that this process is implemented only once before time integration begins.

With the derivative operator $\mathcal{D}$, the decoupled governing equations given in Eqs. (37)-(40) are discretized horizontally with the SEM and vertically with the VFE method, expressed in semidiscrete form as

$$
\frac{d \rho_{i, j}^{\prime}}{d t}+u_{i, j} \hat{\mathbf{D}}_{i, k}^{(\xi, e)} \rho_{k, j}^{\prime}+\rho_{i, j} \hat{\mathbf{D}}_{i, k}^{(\xi, e)} u_{k, j}+\dot{\sigma} \mathcal{D} \rho+\rho \mathcal{D} \dot{\sigma}=0,
$$

$$
\begin{aligned}
& \frac{d u_{i, j}}{d t}+u_{i, j} \hat{\mathbf{D}}_{i, k}^{(\xi, e)} u_{k, j}+\frac{1}{\rho_{i, j}} \hat{\mathbf{D}}_{i, k}^{(\xi, e)} p_{k, j}^{\prime}+f w_{i, j} \\
& +\dot{\sigma} \mathcal{D} u+\frac{1}{\rho} \frac{\partial \sigma}{\partial x} \mathcal{D} p^{\prime}=0 \\
& \frac{d w_{i, j}}{d t}+u_{i, j} \hat{\mathbf{D}}_{i, k}^{(\xi, e)} w_{k, j}+\frac{\rho_{i, j}^{\prime}}{\rho_{i, j}} g-f u_{i, j}+\dot{\sigma} \mathcal{D} w+\frac{1}{\rho} \frac{\partial \sigma}{\partial z} \mathcal{D} p^{\prime}=0
\end{aligned}
$$

and

$$
\frac{d \theta_{i, j}^{\prime}}{d t}+u_{i, j} \hat{\mathbf{D}}_{i, k}^{(\xi, e)} \theta_{k, j}^{\prime}+\dot{\sigma} \mathcal{D}\left(\bar{\theta}+\theta^{\prime}\right)=0,
$$

where the buoyancy and Coriolis terms can be placed either in the horizontal or the vertical direction, since they have no derivatives. In the equations, $\partial \sigma / \partial x$ and $\partial \sigma / \partial z$ denote the Jacobians of the coordinate transformation given in Eqs. (32) and (33), respectively. 


\section{d. Artificial viscosity in terrain-following coordinates}

Most models require some form of viscosity, especially, if the model numerics are nondissipative. This is true for the high-order SEM, since without any added dissipative mechanism, the method is completely nondissipative, which may result in instabilities whenever flows with steep gradients are encountered. The simplest dissipation mechanism is that of a Laplacian operator added to the righthand side of the governing equations given in Eq. (1) as

$$
\frac{\partial \mathbf{q}}{\partial t}=-\mathbf{u} \cdot \nabla \mathbf{q}+S(\mathbf{q})+\nabla \cdot(\mu \nabla \mathbf{q}),
$$

where $\mu$ is the viscosity coefficient. However, this operator is only included in the momentum and temperature equations. For more sophisticated regularizations of these equations, the reader is referred to Marras et al. (2015). Let us now describe how we discretize this operator using terrain-following coordinates for a scalar variable $q$ in the context of the SEM. After applying the Galerkin method and invoking the divergence theorem, the dissipative term given in Eq. (76) becomes

$$
\int_{\Omega_{e}} \psi_{i} \nabla \cdot(\mu \nabla q) d \Omega_{e}=-\int_{\Omega_{e}} \nabla \psi_{i} \cdot(\mu \nabla q) d \Omega_{e},
$$

where the natural boundary condition is assumed for simplicity (i.e., the flux integral at the boundary vanishes). Replacing the gradient operator yields

$$
\begin{aligned}
\int_{\Omega_{e}} \psi_{i} \nabla \cdot(\mu \nabla q) d \Omega_{e} & =-\int_{\tilde{\Omega}_{e}}\left(\tilde{\mathbf{J}} \widetilde{\nabla} \psi_{i}\right) \cdot\left(\mu \tilde{\mathbf{J}}^{\mathscr{T}} \tilde{\nabla} q\right) d \tilde{\Omega}_{e} \\
& =-\int_{\tilde{\Omega}_{e}}\left(\tilde{\nabla} \psi_{i}\right)^{\mathscr{T}} G \tilde{\nabla} q d \tilde{\Omega}_{e},
\end{aligned}
$$

where $\Omega$ and $\tilde{\Omega}$ denote the spaces in the $x-z$ and $x-\sigma$ coordinates, respectively, and $G=\tilde{\mathbf{J}} \mu \tilde{\mathbf{J}}^{\widetilde{T}}$. For the VFE and VFD methods, the gradient operator with respect to $\sigma$ is easily discretized with the derivative operator given in Eq. (71) and the central difference approximation, respectively.

\section{Results and discussion}

The accuracy and efficiency of the vertical discretization schemes in the $\sigma$ terrain-following coordinates, such as the VCG and VFE methods discussed in section 4 , are compared with those of the standard SEM presented in section 3. In addition, the second-order central FDM (Pletcher et al. 2011), used to discretize the vertical terms of the decoupled governing equations, referred to as the VFD method, is also included for comparison. The terrain-following approach is referred to as the 1D method, hereafter, because the horizontal and vertical operators are decoupled into a series of $1 \mathrm{D}$ problems. On the other hand, the standard approach is referred to as the $2 \mathrm{D}$ method, since the discretization of the operators in this approach is handled in a fully coupled, 2D manner, where the curvature introduced by orography is handled by the standard Galerkin machinery.

The test cases studied are: 1 ) the rising thermal bubble (Ahmad and Lindeman 2007; Norman et al. 2011; Li et al. 2013), 2) linear hydrostatic mountain waves (Durran and Klemp 1983; Giraldo and Restelli 2008; Melvin et al. 2010; Weller and Shahrokhi 2014), 3) linear nonhydrostatic mountain waves (Giraldo and Restelli 2008; Melvin et al. 2010; Savre et al. 2016), and 4) Schär mountain waves (Schär et al. 2002; Li et al. 2013; Weller and Shahrokhi 2014; Bao et al. 2015). The first test is performed on a rectangular domain with no orography, while the last three tests, where analytic solutions are also available, are chosen to reveal the effect of orography on the vertical discretization of the $1 \mathrm{D}$ methods. To compare the accuracy of the methods in terms of normalized error norms, the high-order, high-resolution 2D SEM is used as a reference solution. In all test cases described above, the normalized $L_{1}, L_{2}$, and $L_{\infty}$ error norms are defined as

$$
\begin{gathered}
L_{1}(\mathscr{C})=\frac{\sum_{k=1}^{N_{p}}\left|\mathscr{C}_{r}-\mathscr{X}_{c}\right|_{k}}{\sum_{k=1}^{N_{p}}\left|\mathscr{C}_{r}\right|_{k}}, \\
L_{2}(\mathscr{X})=\left[\frac{\sum_{k=1}^{N_{p}}\left(\mathscr{C}_{r}-\mathscr{X}_{c}\right)_{k}^{2}}{\sum_{k=1}^{N_{p}}\left(\mathscr{C}_{r}\right)_{k}^{2}}\right]^{1 / 2},
\end{gathered}
$$

and

$$
L_{\infty}(\mathscr{C})=\frac{\max \left|\mathscr{C}_{r}-\mathscr{C}_{c}\right|_{k}}{\max \left|\mathscr{O}_{r}\right|_{k}},
$$

where $k=1, \ldots, N_{p}$. In Eqs. (79)-(81), $\mathscr{C}$ is the perturbation of either potential temperature, horizontal velocity, or vertical velocity, and the subscripts $r$ and $c$ indicate the reference and computed solutions, respectively. In computing the error norms, the potential temperature perturbation is chosen in the rising thermal bubble test, because it is the variable that exhibits the least amount of influence from acoustic waves in 
nonhydrostatic modeling, where one is typically not interested in resolving the acoustic waves properly. In the SEM, the grid size in the $\mathbf{x}$ direction is defined as $\Delta \mathbf{x}=L_{\mathbf{x}} /\left(N_{\mathbf{x}} N_{e, \mathbf{x}}\right)$, where $L_{\mathbf{x}}$ and $N_{e, \mathbf{x}}$ are the domain length and number of elements in the $\mathbf{x}$ direction, respectively. In addition, $N_{\mathbf{x}}$ is the polynomial order along the $\mathbf{x}$ direction. Because of the nonuniform LGL points in the elements, the minimum grid size is actually smaller than $\Delta \mathbf{x}$ in the high-order SEM. In this study, three different grid resolutions are employed for each test to evaluate the grid dependency of the 1D methods with the $L_{2}$ and $L_{\infty}$ error norms. The detailed equations for initializing a uniformly stratified flow under a constant Brunt-Väisälä frequency $\left(N_{f}\right)$ can be found in Giraldo and Restelli (2008).

\section{a. Rising thermal bubble}

As described in Norman et al. (2011), this test consists of a neutral atmosphere with the addition of a warm bubble in a rectangular domain of size $(x, z) \in(0,20) \times$ $(0,10) \mathrm{km}^{2}$. Initially, the perturbation given as the warm bubble is added to the constant potential temperature field of $\bar{\theta}=300 \mathrm{~K}$ in a quiescent flow of $\bar{u}=\bar{w}=0 \mathrm{~m} \mathrm{~s}^{-1}$, where the initial potential temperature perturbation is defined as

$$
\theta^{\prime}(x, z, 0)=\theta_{c} \max \left(0,1-r / r_{c}\right),
$$

where $r=\sqrt{\left(x-x_{c}\right)^{2}+\left(z-z_{c}\right)^{2}}$. In the equation, the physical parameters are set to $\theta_{c}=2 \mathrm{~K}, x_{c}=10 \mathrm{~km}, z_{c}=$ $2 \mathrm{~km}$ and $r_{c}=2 \mathrm{~km}$ (Ahmad and Lindeman 2007; Norman et al. 2011; Li et al. 2013). The no-flux boundary condition (Kelly and Giraldo 2012) is imposed on all sides of the rectangular domain. In this test, the 2D SEM is performed with $N=5, N_{e, x}=60$ and $N_{e, z}=30$ (i.e., $\Delta x=\Delta z=0.067 \mathrm{~km})$. For the $1 \mathrm{D}$ methods, the same horizontal grid is used, while the uniform grids of $N_{\sigma}=$ $2, N_{e, \sigma}=75$ for the VCG method and $N_{e, \sigma}=150$ for the VFD and VFE methods are employed in the vertical direction. The time step is set to $\Delta t=0.1 \mathrm{~s}$, which corresponds to $C_{h}=C_{v}=0.89$ for the $2 \mathrm{D} \mathrm{SEM}$ and $C_{h}=$ $0.89, C_{v}=0.52$ for the $1 \mathrm{D}$ methods. Because all the methods produce large oscillations around the bubble, resulting in noisy solutions after about $900 \mathrm{~s}$, the dissipation term given in Eq. (76) is added to the governing equations to stabilize the solution with $\mu=4 \mathrm{~m}^{2} \mathrm{~s}^{-1}$, which is determined to minimize its effect on the comparison of accuracy.

Since the bubble is warmer than its surrounding atmosphere, it rises upward as shown in Fig. 2 , in which the potential temperature and vertical velocity perturbations obtained from the VFE method are captured at $1000 \mathrm{~s}$. The flow patterns of the potential temperature
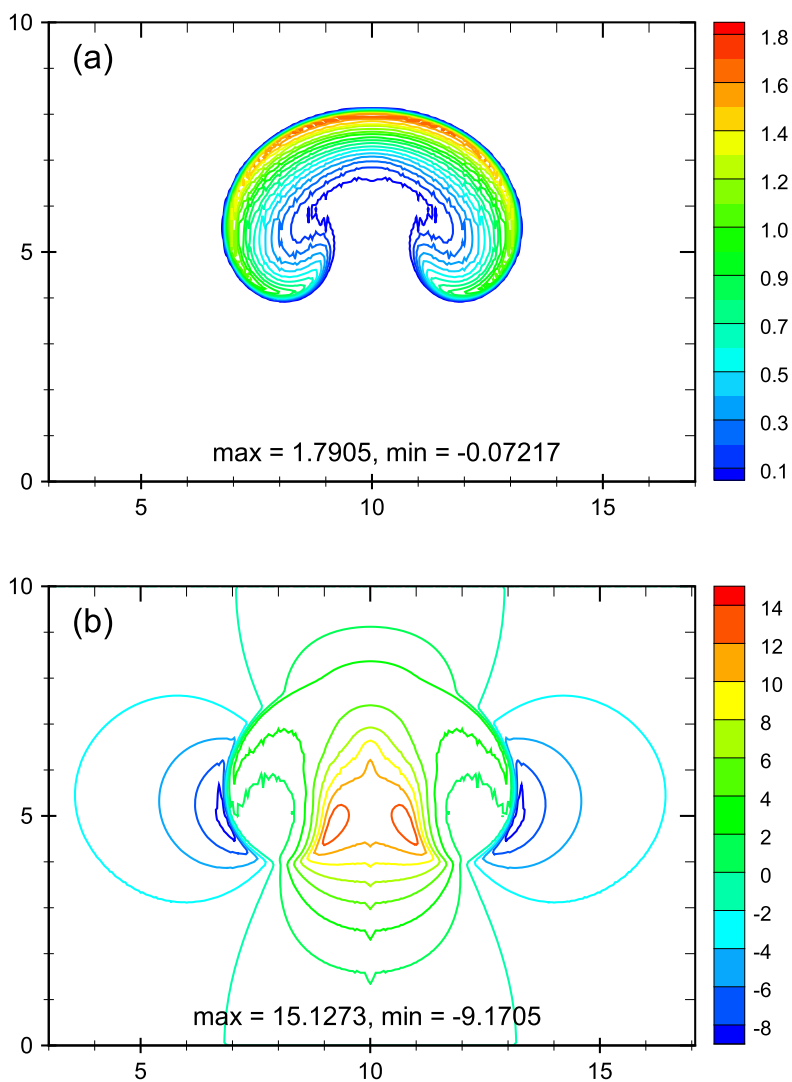

FIG. 2. Rising thermal bubble at $1000 \mathrm{~s}$ : (a) potential temperature perturbation and (b) vertical velocity perturbation of the 1D VFE method with $\Delta x=\Delta z=0.067 \mathrm{~km}$. The negligible positive and negative values (close to zero) around the bubble are removed for a smooth representation in potential temperature perturbation. Spatial units are in kilometers.

and vertical velocity perturbations obtained from the $1 \mathrm{D}$ methods are nearly identical to those obtained from the 2D SEM, except for the VFD method in which large oscillations are observed along the bottom of the bubble (not shown). Moreover, the shape and location of the bubble in the figures are quite similar to the results of Norman et al. (2011), where $\Delta x=\Delta z=0.125 \mathrm{~km}$ were used. At this grid resolution, the maximum values of the potential temperature and vertical velocity perturbations in Norman et al. (2011) and Ahmad and Lindeman (2007) are close to those in Fig. 2. However, the maximum potential temperature perturbation of Fig. 2a is slightly lower than that of Li et al. (2013) at similar grid resolutions.

More detailed comparisons are performed with the normalized $L_{1}, L_{2}$, and $L_{\infty}$ error norms of the potential temperature and vertical velocity perturbations and the wall-clock time, given in Table 1 . In the table, the 2D SEM with the grid of $N=5, N_{e, x}=120$ and $N_{e, z}=60$ $(\Delta x=\Delta z=0.033 \mathrm{~km})$, and $\mu=5 \mathrm{~m}^{2} \mathrm{~s}^{-1}$ is adopted as a 
TABLE 1. Comparison of the accuracy and efficiency of vertical discretization methods for the rising thermal bubble test with $\Delta x=\Delta z=$ $0.067 \mathrm{~km}$. The fifth-order 2D SEM with $\Delta x=\Delta z=0.033 \mathrm{~km}$ is used as a reference solution. WT denotes the wall-clock time in seconds.

\begin{tabular}{|c|c|c|c|c|c|c|c|}
\hline \multirow[b]{2}{*}{ Methods } & \multicolumn{3}{|c|}{$\theta^{\prime}(\mathrm{K})$} & \multicolumn{3}{|c|}{$w^{\prime}\left(\mathrm{m} \mathrm{s}^{-1}\right)$} & \multirow[b]{2}{*}{ WT (s) } \\
\hline & $L_{1}$ & $L_{2}$ & $L_{\infty}$ & $L_{1}$ & $L_{2}$ & $L_{\infty}$ & \\
\hline 2D SEM & 0.05763 & 0.05030 & 0.08786 & 0.00756 & 0.01618 & 0.06037 & 1160.5 \\
\hline 1D VCG & 0.04585 & 0.04846 & 0.16173 & 0.00753 & 0.01672 & 0.06049 & 2995.2 \\
\hline 1D VFD & 0.17229 & 0.22161 & 0.40127 & 0.01845 & 0.04444 & 0.15901 & 933.5 \\
\hline 1D VFE & 0.03635 & 0.03348 & 0.07860 & 0.00688 & 0.01539 & 0.05972 & 2882.3 \\
\hline
\end{tabular}

reference solution to be compared with the $1 \mathrm{D}$ and $2 \mathrm{D}$ approaches in the accuracy of spatial discretization. The table reveals that the 1D VFE method produces the smallest $L_{1}, L_{2}$, and $L_{\infty}$ error norms in the potential temperature perturbation and yields slightly higher accuracy than the 2D SEM and 1D VCG in the vertical velocity perturbation. On the other hand, the $1 \mathrm{D}$ VFD method provides the error norms much larger than the other methods, as expected. The wall-clock time of the $1 \mathrm{D}$ VCG method is approximately $158 \%$ larger than that of the 2D SEM, because it uses the exact integration of the Gaussian quadrature, which requires the inversion of a vertical mass matrix. The 1D VFD method runs approximately $20 \%$ faster than the $2 \mathrm{D}$ SEM, since the discretization of the vertical derivatives with the central FDM used in this study is quite straightforward. This test requires additional computation for the artificial viscosity, as given in Eq. (76). In the 1D VFE method, expressed by matrix multiplication, the computation of a second derivative operator is more expensive than that of the other methods. This causes the wall-clock time of the 1D VFE method to be much greater than that of the 2D SEM by $148 \%$. The computational time of the VFE method might be reduced without loss of overall accuracy if the second derivative operator is discretized with the central FDM in the framework of the VFE method. This reduces the computational time to $1897 \mathrm{~s}$, about a $34 \%$ reduction, while $L_{1}=0.03935, L_{2}=0.03586, L_{\infty}=0.07875$ for the potential temperature perturbation and $L_{1}=0.00692$, $L_{2}=0.01545, L_{\infty}=0.05982$ for the vertical velocity perturbation.

The normalized $L_{2}$ and $L_{\infty}$ error norms of the vertical velocity perturbation obtained from the $1 \mathrm{D}$ and $2 \mathrm{D}$ methods are compared at three different grid resolutions to investigate their grid dependency, given in Fig. 3, where the viscosity coefficient is increased by $0.5 \mathrm{~m}^{2} \mathrm{~s}^{-1}$ from 3.5 to $4.5 \mathrm{~m}^{2} \mathrm{~s}^{-1}$ as the grid resolution increases. In the coarsest grid, the VFE method shows the better accuracy than the other methods. As the grid resolution increases, however, the solutions of the 2D SEM, 1D VFE and VCG gradually converge to similar values. On the other hand, the VFD method is significantly worse in accuracy than the other methods at all the grid resolutions. With the vertical velocity perturbation, the $L_{2}$ and $L_{\infty}$ error norms of the 2D SEM converge at 3.9th- and 3.5th-order rates, respectively. For the 1D methods, the convergence of the 1D VCG method is computed as 3.5th and 3.9th order in both the error norms, whereas the solution of 1D VFE method converges with the accuracies of 3.1th and 2.2th order, respectively. The 1D VFD yields the slower convergence rates, computed as the $L_{2}$ and $L_{\infty}$ error norms of 1.5 th and 1.1th order, respectively. In this
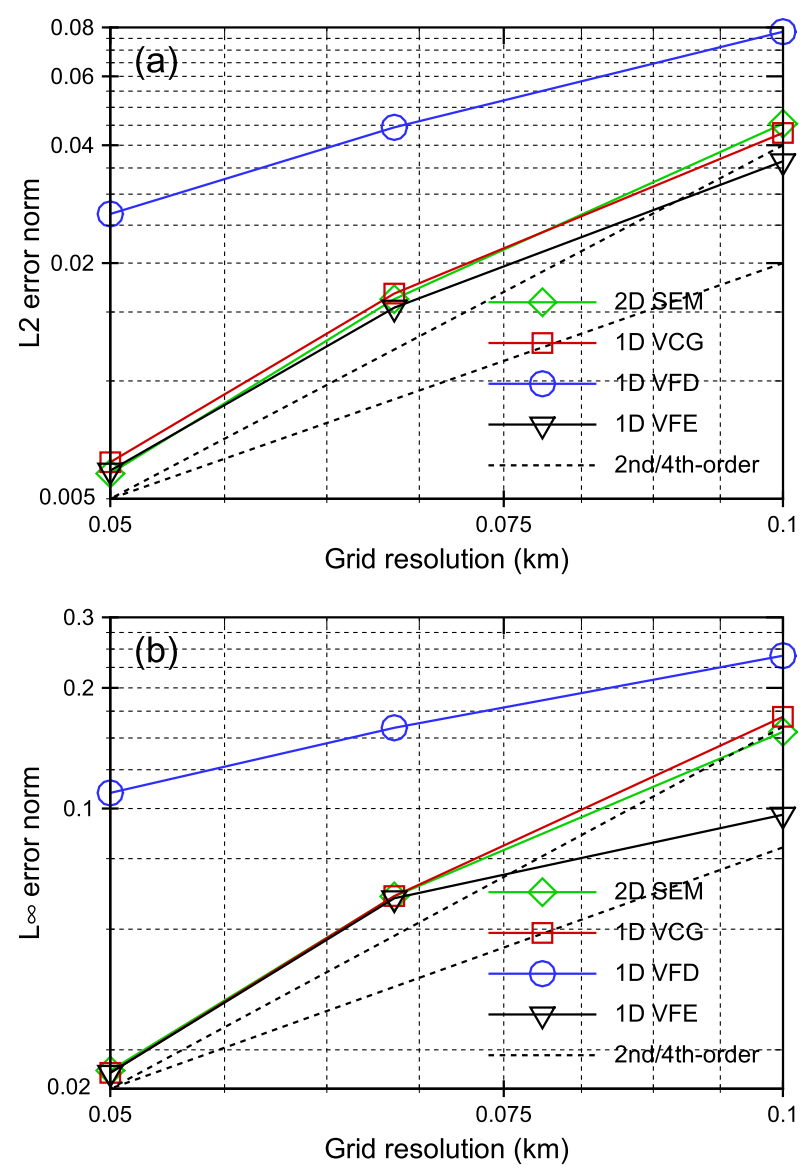

FIG. 3. Grid convergence of vertical discretization methods in the normalized (a) $L_{2}$ and (b) $L_{\infty}$ error norms of vertical velocity perturbation for the test of rising thermal bubble. The axes are in a $\log$ scale. 

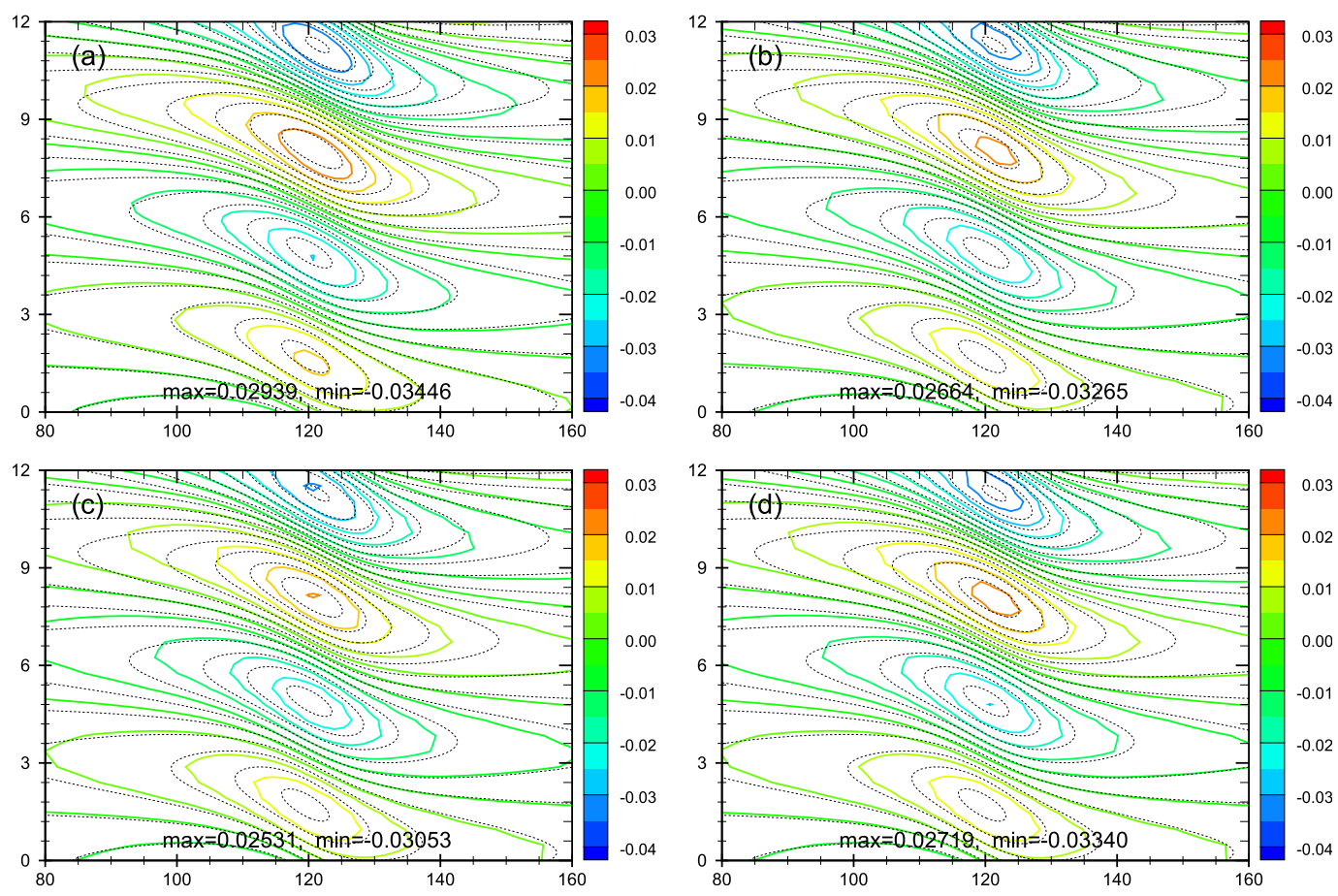

FIG. 4. Horizontal velocity perturbation of vertical discretization methods at $\bar{u} t / a_{c}=60$ for the test of linear hydrostatic mountain waves with $\Delta x=1.2 \mathrm{~km}$ and $\Delta z=0.24 \mathrm{~km}$ : (a) 2D SEM, (b) 1D VCG, (c) 1D VFD, and (d) $1 \mathrm{D}$ VFE. The dotted lines indicate an analytic solution, where its maximum and minimum values are 0.0327 and $-0.04057 \mathrm{~m} \mathrm{~s}^{-1}$, respectively. Spatial units are in kilometers.

test, the convergence rates of all the methods expressed in the $L_{2}$ and $L_{\infty}$ error norms are lower than the spatial discretization accuracy. The loss of spatial accuracy was also observed in the rising thermal bubble test due to the use of vertical viscosity (Guerra and Ullrich 2016) and in inertia gravity wave test due to a splitting error (Bao et al. 2015). In the rising thermal bubble test of Weller and Shahrokhi (2014), the convergence rate with secondorder finite volumes was computed as second order at $400 \mathrm{~s}$ and then reduced to first order at $1000 \mathrm{~s}$ due to the insufficient resolution of sharp gradients.

In addition to Table 1 , the $1 \mathrm{D}$ VCG method with $N_{\sigma}=$ 5 and $N_{e, \sigma}=30$ in the vertical direction is compared with the 2D SEM, where it uses the LGL grid in both directions but is coupled. The results reveal that the $1 \mathrm{D}$ VCG method yields exactly the same maximum and minimum values as the 2D SEM and zero error norms (not shown). This fact confirms that, with no orography, both methods in the same grid are not only mathematically equivalent but also numerically identical.

\section{b. Linear hydrostatic mountain waves}

This mountain wave test involves the steady-state solution of a hydrostatically balanced flow, induced by the topography of a small mountain. It shows approximately linear and hydrostatic behavior because the mountain is low and wide (Durran and Klemp 1983). The horizontal and vertical grids are set to be $N_{e, x}=40$ and $N_{e, z}=20$, respectively, with $N=5$ for the 2D SEM, whereas in the $1 \mathrm{D}$ methods, the vertical grid is generated with $N_{\sigma}=2, N_{e, \sigma}=50$ for the VCG method to be uniform and $N_{e, \sigma}=100$ for the VFD and VFE methods, so that $\Delta x=1.2 \mathrm{~km}$ and $\Delta z=0.24 \mathrm{~km}$. A time step of $\Delta t=0.4 \mathrm{~s}$ is used for this grid resolution, which corresponds to $C_{h}=0.19, C_{v}=0.9$ for the 2D SEM and $C_{h}=0.19, C_{v}=0.53$ for the $1 \mathrm{D}$ methods.

The idealized test with linear hydrostatic waves, triggered by a $1-\mathrm{m}$ low mountain in an isothermal atmosphere, is performed to compare the accuracy of the 1D and 2D vertical discretization methods against the fifthorder 2D SEM with $\Delta x=0.53 \mathrm{~km}$ and $\Delta z=0.12 \mathrm{~km}$. The bell-shaped mountain is described on the surface with a height of $h_{0}=1 \mathrm{~m}$ and a half-width of $a_{c}=10 \mathrm{~km}$ in the domain of $(x, z) \in[0,240] \times[0,24] \mathrm{km}^{2}$, given as

$$
h(x)=h_{0} \frac{a_{c}^{2}}{\left(x-x_{c}\right)^{2}+a_{c}^{2}},
$$

where the mountain center is located at $x_{c}=120 \mathrm{~km}$ (Durran and Klemp 1983; Giraldo and Restelli 2008). To initialize a uniformly stratified atmosphere, the flow field is set to $\bar{u}=20 \mathrm{~m} \mathrm{~s}^{-1}, \bar{w}=0 \mathrm{~m} \mathrm{~s}^{-1}, \bar{\theta}=250 \mathrm{~K}$, and 


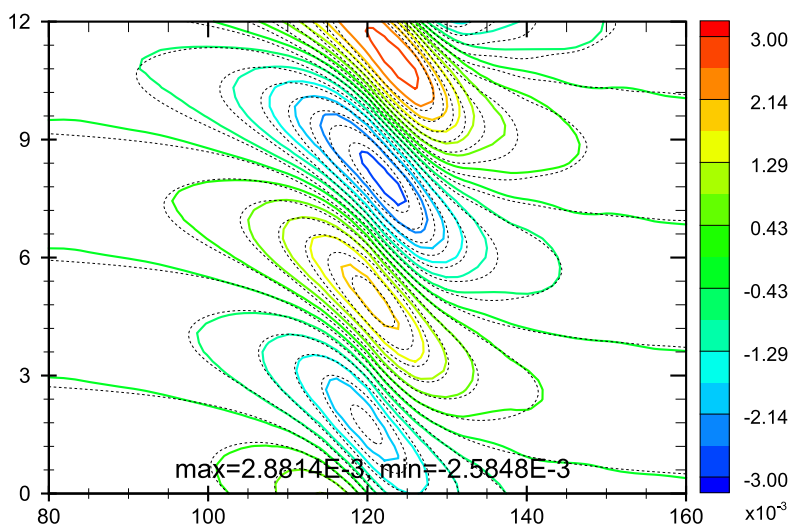

FIG. 5. Vertical velocity perturbation of the 1D VFE method at $\bar{u} t / a_{c}=60$ for the test of linear hydrostatic mountain waves with $\Delta x=1.2 \mathrm{~km}$ and $\Delta z=0.24 \mathrm{~km}$. The dotted lines indicate an analytic solution, where its maximum and minimum values are $4.1629 \times 10^{-3}$ and $-3.3844 \times 10^{-3} \mathrm{~m} \mathrm{~s}^{-1}$, respectively. Spatial units are in kilometers.

$N_{f}=0.0196 \mathrm{~s}^{-2}$. For the boundary conditions, the sponge layers formulated in Eqs. (26) and (27) are applied to the left, right and top sides of the domain in which their widths are 20,20 , and $12 \mathrm{~km}$, respectively, and the sponge coefficients are set to $\tau_{\text {lat }}=0.1$ and $\tau_{\text {top }}=$ 0.2 . The no-flux boundary condition is imposed on the bottom surface.

The horizontal velocity field around the mountain at $\bar{u} t / a_{c}=60$ is shown in Fig. 4 within the domain of $(x, z) \in$ $[80,160] \times[0,12] \mathrm{km}^{2}$, whereby the linear analytic solution (Durran and Klemp 1983) is also given in each figure, indicated by dotted lines, for easier comparison. In the figures, the horizontal velocity perturbations of the 2D SEM and the 1D methods are similar to the analytic solution. Moreover, the flow pattern and quantity of the horizontal velocity perturbations are in good agreement with the numerical results of Durran and Klemp (1983) and Giraldo and Restelli (2008). In the work of Durran and Klemp (1983), where $\Delta x=$ $2 \mathrm{~km}$ and $\Delta z=0.2 \mathrm{~km}$ were used, the horizontal velocity perturbation was in the range of 0.024 to $-0.024 \mathrm{~m} \mathrm{~s}^{-1}$, while that of the VFE method is between 0.027 and $-0.033 \mathrm{~m} \mathrm{~s}^{-1}$, as given in Fig. 4 .
The flow pattern of the vertical velocity perturbation is similar to the analytic solution as well as to that of Melvin et al. (2010) and Weller and Shahrokhi (2014). Figure 5 shows this result obtained with the VFE method with $\Delta x=1.2 \mathrm{~km}$ and $\Delta z=0.24 \mathrm{~km}$. In Fig. 5, the maximum and minimum vertical velocity perturbations are computed as $2.881 \times 10^{-3}$ and $-2.585 \times 10^{-3} \mathrm{~m} \mathrm{~s}^{-1}$, respectively. To compare their accuracy quantitatively, the normalized error norms of the horizontal and vertical velocity perturbations are listed in Table 2 . The results in the table show that the 2D SEM yields more accurate solutions than the 1D methods. Among the 1D methods, the VFE method achieves the smallest error norms for both the horizontal and vertical velocity perturbations, whereas the second-order VFD method produces the worst results regarding accuracy, as pointed out in the previous test. For efficiency, the computational time of the VCG method is computed to be $82 \%$ greater than that of the 2D SEM, while the 1D VFD method runs fastest by $9 \%$, which is consistent with the previous test case. For the VFE method, the rate of increase in the computational time is significantly reduced to $37 \%$ because no artificial viscosity is used in this test. In Fig. 6, the convergence rates of the vertical velocity perturbation are given in terms of the normalized $L_{2}$ and $L_{\infty}$ error norms versus the grid resolutions. Unlike the rising thermal bubble test, the 2D SEM yields higher accurate results than the 1D methods at all the grid resolutions, converged with the accuracies of 2.2 th and 2.7 th order, respectively. This might be due to the coordinate transformation error of the 1D methods on orography. Among the 1D methods, the VFE method produces higher accuracy than the VCG or VFD method at all the grid resolutions, converged at 2.1th order in both the error norms. Furthermore, the VCG and VFD methods show the convergence rates between 1.9th and 2.2th order.

\section{c. Linear nonhydrostatic mountain waves}

The second test with the mountain waves is considered as the steady-state solution of a linear nonhydrostatic flow (Giraldo and Restelli 2008; Melvin et al. 2010; Savre et al. 2016) because it uses a narrower

TABLE 2. Comparison of the accuracy and efficiency of vertical discretization methods for the test of linear hydrostatic mountain waves with $\Delta x=1.2$ and $\Delta z=0.24 \mathrm{~km}$. The fifth-order 2D SEM with $\Delta x=0.53 \mathrm{~km}$ and $\Delta z=0.12 \mathrm{~km}$ is used as a reference solution.

\begin{tabular}{|c|c|c|c|c|c|c|c|}
\hline \multirow[b]{2}{*}{ Methods } & \multicolumn{3}{|c|}{$u^{\prime}\left(\mathrm{m} \mathrm{s}^{-1}\right)$} & \multicolumn{3}{|c|}{$w^{\prime}\left(\mathrm{m} \mathrm{s}^{-1}\right)$} & \multirow[b]{2}{*}{ WT (s) } \\
\hline & $L_{1}$ & $L_{2}$ & $L_{\infty}$ & $L_{1}$ & $L_{2}$ & $L_{\infty}$ & \\
\hline 2D SEM & 0.04599 & 0.05016 & 0.10606 & 0.10239 & 0.10051 & 0.12335 & 4620.1 \\
\hline 1D VCG & 0.16158 & 0.17515 & 0.19786 & 0.17705 & 0.20475 & 0.29255 & 8403.2 \\
\hline 1D VFD & 0.17496 & 0.18952 & 0.22690 & 0.18966 & 0.21872 & 0.30134 & 4217.0 \\
\hline 1D VFE & 0.13930 & 0.15208 & 0.17649 & 0.16119 & 0.18548 & 0.26902 & 6331.1 \\
\hline
\end{tabular}



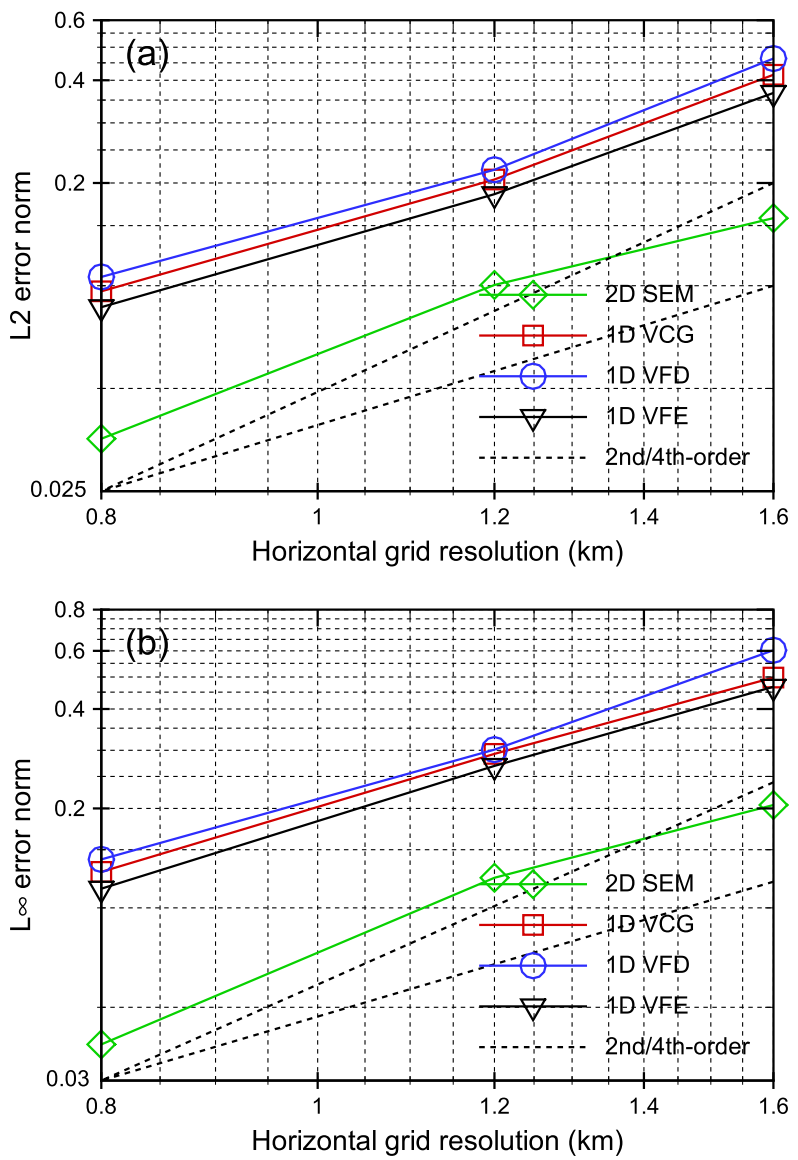

FIG. 6. Grid convergence of vertical discretization methods in the normalized (a) $L_{2}$ and (b) $L_{\infty}$ error norms of vertical velocity perturbation for the test of linear hydrostatic mountain waves. The axes are in a log scale.

mountain than the hydrostatic mountain. This case uses the same topographical mountain profile as the first mountain test, given in Eq. (83), but with $a_{c}=1 \mathrm{~km}$. The physical configuration is given by $\bar{u}=10 \mathrm{~m} \mathrm{~s}^{-1}$, $\bar{w}=0 \mathrm{~m} \mathrm{~s}^{-1}, \bar{\theta}=280 \mathrm{~K}$, and $N_{f}=0.01 \mathrm{~s}^{-2}$, and the computational domain is confined by $(x, z) \in[0,144] \times$ $[0,30] \mathrm{km}^{2}$, where $x_{c}=72 \mathrm{~km}$. The boundary conditions are the same as in the hydrostatic mountain test, where the widths of the sponge layer on the left, right and top of the domain are set to 20,20 , and $17 \mathrm{~km}$, respectively, and their coefficients are $\tau_{\text {lat }}=\tau_{\text {top }}=0.1$.

The horizontal velocity perturbations obtained from the $2 \mathrm{D}$ SEM and the $1 \mathrm{D}$ methods at $5 \mathrm{~h}$ are shown in Fig. 7 within the domain $(x, z) \in[60,100] \times[0,12] \mathrm{km}^{2}$, where the analytic solution is also added to each figure, indicated by the dotted lines. In the figures, the horizontal grid is set to $N_{x}=5, N_{e, x}=80$ for all the methods, while the vertical grid is employed with $N_{z}=5, N_{e, z}=30$ for the 2D SEM, $N_{\sigma}=2, N_{e, \sigma}=75$ for the $1 \mathrm{D}$ VCG, and $N_{e, \sigma}=150$ for the 1D VFD and VFE methods, resulting in $\Delta x=0.36 \mathrm{~km}$ and $\Delta z=0.2 \mathrm{~km}$. The time step is set to $\Delta t=0.3 \mathrm{~s}$ for this grid resolution, so that $C_{h}=0.49, C_{v}=$ 0.86 for the 2D SEM and $C_{h}=0.49, C_{v}=0.5$ for the $1 \mathrm{D}$ methods. Figure 7 shows that the horizontal velocity perturbations of the $1 \mathrm{D}$ methods are qualitatively similar to those for the 2D SEM. Moreover, they are close to the analytic solution near the mountain side, but rather different for the upper-right side, due to the sponge layers, as pointed out in Giraldo and Restelli (2008) and Savre et al. (2016). Unlike the horizontal velocity perturbation, the distributions of the vertical velocity perturbation, obtained from all the methods, are very close to the analytic and reference solutions. Figure 8 shows the contour plot of the vertical velocity perturbation, obtained with the VFE method with $\Delta x=0.36 \mathrm{~km}$ and $\Delta z=0.2 \mathrm{~km}$. Its flow pattern is in good agreement with the results of Giraldo and Restelli (2008), Melvin et al. (2010) and Savre et al. (2016).

For quantitative details, the comparison of the accuracy of the 2D SEM and the 1D methods against the fifth-order 2D SEM with $\Delta x=0.18 \mathrm{~km}$ and $\Delta z=0.1 \mathrm{~km}$ is given in Table 3. As in the previous mountain wave test, the $L_{1}, L_{2}$, and $L_{\infty}$ error norms of the 2D SEM are the smallest in both the horizontal and vertical velocity perturbations. For the 1D methods in this test, the 1D VFE method also provides smaller error norms than the 1D VCG or VFD methods. Regarding efficiency, this test exhibits a similar tendency in the wall-clock time as in the previous tests, indicating that the 1D VCG and VFE methods are $89 \%$ and $54 \%$ more expensive, respectively, than the 2D SEM. Similar to the grid convergence of the hydrostatic mountain wave test, the 2D SEM yields smaller $L_{2}$ and $L_{\infty}$ error norms than the other $1 \mathrm{D}$ methods at all the grid resolutions, converged with the second- and 2.4th-order rates, respectively, as shown in Fig. 9. The convergence rates of the $1 \mathrm{D}$ methods in terms of the $L_{2}$ error norm are in the range of 2.7th to 3.1th order, while the $L_{\infty}$ error norms converge with the accuracies of 3.5th, 3.1th, and 3.8th order in the order in Fig. 9.

\section{d. Schär mountain waves}

The last case is the Schär mountain test (Schär et al. 2002; Li et al. 2013; Weller and Shahrokhi 2014; Bao et al. 2015), where the terrain profile is more complex and higher than that of the previous mountain tests. The profile is constructed by a $h_{0}$-height bell-shaped mountain superposed with a small-scale cosine function (Schär et al. 2002), given as

$$
h(x)=h_{0} \exp \left(-\frac{x^{2}}{a_{c}^{2}}\right) \cos ^{2}\left(\frac{\pi x}{\lambda}\right),
$$

where $h_{0}=0.25 \mathrm{~km}, a_{c}=5 \mathrm{~km}$, and $\lambda=4 \mathrm{~km}$ in the computational domain of $(x, z) \in[-25,25] \times[0,21] \mathrm{km}^{2}$. 

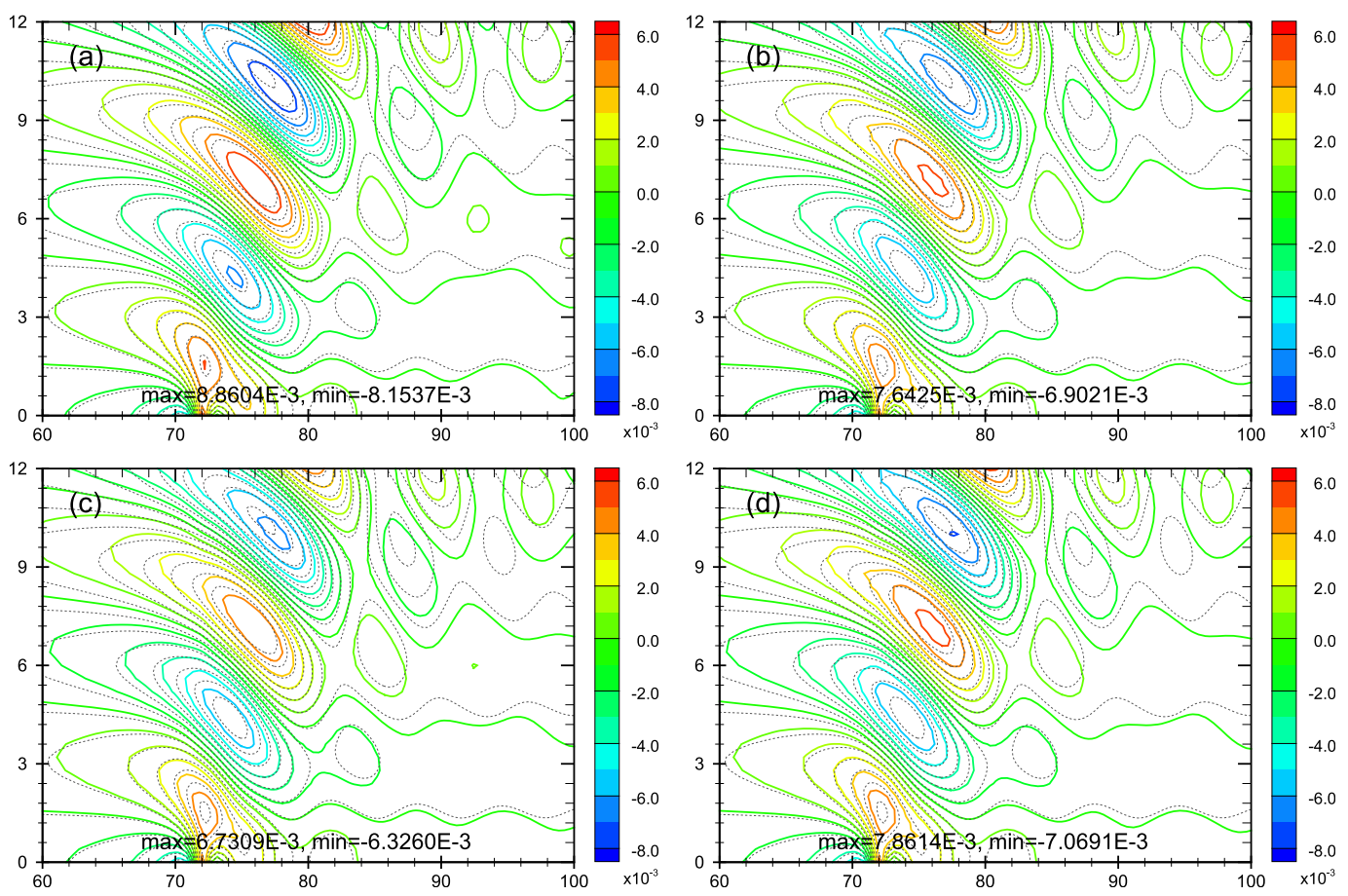

FIG. 7. Horizontal velocity perturbation of vertical discretization methods at $5 \mathrm{~h}$ for the test of linear nonhydrostatic mountain waves with $\Delta x=0.36 \mathrm{~km}$ and $\Delta z=0.2 \mathrm{~km}$ : (a) 2D SEM, (b) 1D VCG, (c) 1D VFD, and (d) 1D VFE. The dotted lines indicate an analytic solution, where its maximum and minimum values are $9.6621 \times$ $10^{-3}$ and $-8.2482 \times 10^{-3} \mathrm{~m} \mathrm{~s}^{-1}$, respectively. Spatial units are in kilometers.

The initial condition is set to $\bar{u}=10 \mathrm{~m} \mathrm{~s}^{-1}, \bar{w}=0 \mathrm{~m} \mathrm{~s}^{-1}$, $\bar{\theta}=280 \mathrm{~K}$, and $N_{f}=0.01 \mathrm{~s}^{-2}$. As in the previous mountain tests, the no-flux boundary condition is applied to the bottom surface and the nonreflecting boundary condition is imposed on the top and lateral boundaries of the domain, where the width of the sponge layer is set to $11 \mathrm{~km}$ for those boundaries and the sponge coefficients are $\tau_{\text {lat }}=\tau_{\text {top }}=0.1$.

The Schär mountain test is performed until $t=10$ hours at which the solution converges to a steady state, as shown in Fig. 10. For the 2D SEM in the figures, the horizontal and vertical grids are constructed with $N_{e, x}=$ 40 and $N_{e, z}=28$, respectively, with $N=5$, so that $\Delta x=$ $0.25 \mathrm{~km}$ and $\Delta z=0.15 \mathrm{~km}$. For the $1 \mathrm{D}$ methods, the vertical grid of $N_{\sigma}=2, N_{e, \sigma}=70$ is employed in the VCG method, while $N_{\sigma}=140$ is set in the VFD and VFE methods. The time step of the explicit SSP-RK scheme is set to $\Delta t=0.22 \mathrm{~s}$, which results in $C_{h}=0.52$, $C_{v}=0.84$ for the 2D SEM and $C_{h}=0.52, C_{v}=0.49$ for the $1 \mathrm{D}$ methods. Figure 10 denotes the vertical velocity perturbation obtained from the $1 \mathrm{D}$ and $2 \mathrm{D}$ vertical discretization methods. In these figures, a distorted wave pattern on the upper levels does not appear, which is induced by numerical errors in the horizontal pressure gradient over steep topography in the $\sigma$ coordinate (Sundqvist 1975; Haney 1991; Schär et al. 2002; Klemp et al. 2003). The distributions of the vertical velocity perturbation obtained from the 1D methods are similar to the 2D SEM and analytic solutions as well as the results in the literature (Schär et al. 2002; Li et al. 2013; Weller and Shahrokhi 2014; Bao et al. 2015). Moreover, the horizontal velocity perturbation, obtained from the VFE method, is plotted in Fig. 11, where the flow pattern

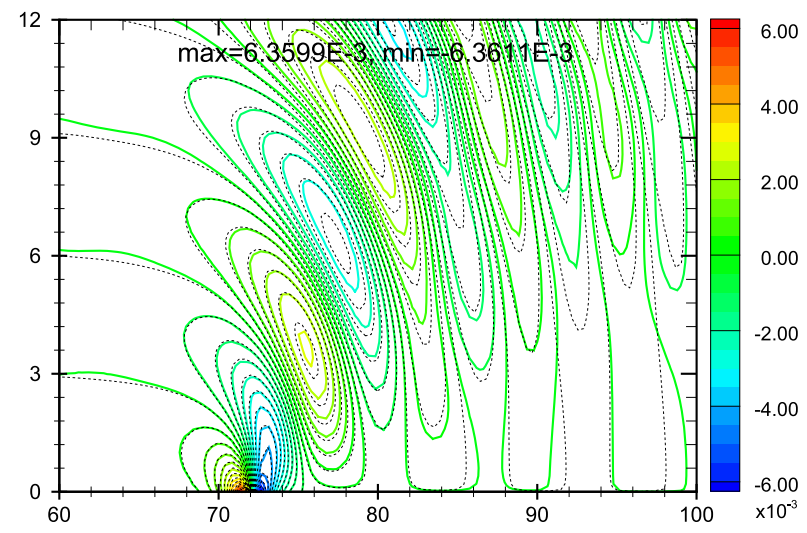

FIG. 8. Vertical velocity perturbation of the 1D VFE method at $5 \mathrm{~h}$ for the test of linear nonhydrostatic mountain waves with $\Delta x=$ $0.36 \mathrm{~km}$ and $\Delta z=0.2 \mathrm{~km}$. The dotted lines indicate an analytic solution, where its maximum and minimum values are $6.2341 \times$ $10^{-3}$ and $-6.2341 \times 10^{-3} \mathrm{~m} \mathrm{~s}^{-1}$, respectively. Spatial units are in kilometers. 
TABLE 3. Comparison of the accuracy and efficiency of vertical discretization methods for the test of linear nonhydrostatic mountain waves with $\Delta x=0.36 \mathrm{~km}$ and $\Delta z=0.2 \mathrm{~km}$. The fifth-order 2D SEM with $\Delta x=0.18 \mathrm{~km}$ and $\Delta z=0.1 \mathrm{~km}$ is used as a reference solution.

\begin{tabular}{lccccccr}
\hline \hline & \multicolumn{3}{c}{$u^{\prime}\left(\mathrm{m} \mathrm{s}^{-1}\right)$} & & \multicolumn{3}{c}{$w^{\prime}\left(\mathrm{m} \mathrm{s}^{-1}\right)$} \\
\cline { 2 - 4 } Methods & $L_{1}$ & $L_{2}$ & $L_{\infty}$ & & $L_{1}$ & $L_{2}$ & $L_{\infty}$ \\
\hline 2D SEM & 0.14553 & 0.12338 & 0.12544 & & 0.14448 & 0.13636 & 0.09340 \\
1D VCG & 0.18258 & 0.17369 & 0.30171 & 0.18112 & 0.18352 & 0.15106 & 22867.3 \\
1D VFD & 0.21427 & 0.20902 & 0.33934 & 0.21251 & 0.21483 & 0.18607 & 11046.2 \\
1D VFE & 0.16950 & 0.15755 & 0.27217 & 0.16837 & 0.16885 & 0.13197 & 18660.4 \\
\hline
\end{tabular}

is similar to the analytic solution and the results of $\mathrm{Li}$ et al. (2013) and Bao et al. (2015). In the work of Bao et al. (2015), the contour levels of the horizontal and vertical velocity perturbations were in the range of 2 to $-2 \mathrm{~m} \mathrm{~s}^{-1}$, which are close to those of Figs. 10 and 11 .

A further comparison is given in Table 4, where the reference solution used for evaluating the error norms is obtained from the fifth-order 2D SEM with $\Delta x=0.11 \mathrm{~km}$ and $\Delta z=0.07 \mathrm{~km}$. Among the $1 \mathrm{D}$ methods, the 1D VFE method produces the smallest error norms in both the horizontal and vertical velocity perturbations, while the 1D VFD shows the worst accuracy. The 1D and 2D methods provide efficiencies similar to those for the previous mountain tests. Compared with the 2D SEM, the computational times of the 1D VCG and VFE methods are increased by $100.4 \%$ and $56.9 \%$, respectively, whereas that of the 1D VFD method is reduced by $6.9 \%$. This test also provides the grid resolution dependency, as shown in Fig. 12. As the grid resolution increases, the $L_{2}$ and $L_{\infty}$ error norms of the 2D SEM converges with the 1.1th- and 0.9th-order rates, respectively, while the $1 \mathrm{D}$ methods yield approximately 1.2 th-order convergence in the $L_{2}$ error norm and 1.7th order in the $L_{\infty}$ error norm. Figure $12 \mathrm{dem}$ onstrates that the VFE method achieves the smallest $L_{2}$ error norms at all grid resolutions among the 1D methods. Unlike the previous tests, this test shows that the convergence rates of all the methods are significantly lower than the order of discretization accuracy.

\section{Conclusions}

The vertical discretization of two-dimensional nonhydrostatic equations, decoupled in height-based $x-\sigma$ terrain-following coordinates, is formulated with the finite element method based on either Lagrange or b-spline polynomial functions. This approach is derived from a fully coupled high-order spectral element model that handles nonconvex geometries, which occurs when resolving flow over mountains. The main reason to transform $x-z$ coordinates to $x-\sigma$ terrain-following coordinates is to more easily accommodate existing physical parameterizations in a vertical grid by decoupling the governing equations into the horizontal and vertical components. Therefore, in this study, the formulations of the governing equations, discretized in the $x-\sigma$ coordinates, are provided in the forms of VCG and VFE for the vertical terms, while the horizontal terms are discretized with high-order spectral elements on an LGL grid.

To verify the 1D decoupling methods and assess their performance in terms of accuracy and efficiency, four idealized tests are performed in the $x-\sigma$ terrain-following
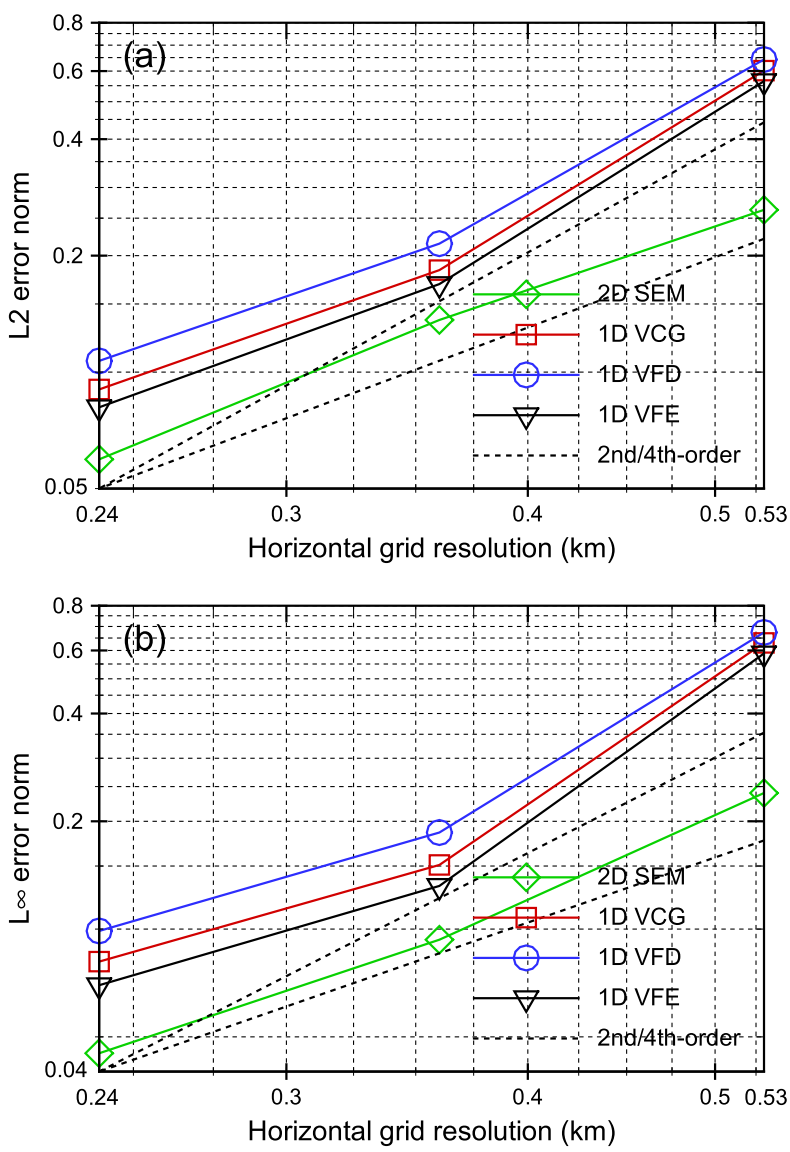

FIG. 9. Grid convergence of vertical discretization methods in the normalized (a) $L_{2}$ and (b) $L_{\infty}$ error norms of vertical velocity perturbation for the test of linear nonhydrostatic mountain waves. The axes are in a log scale. 

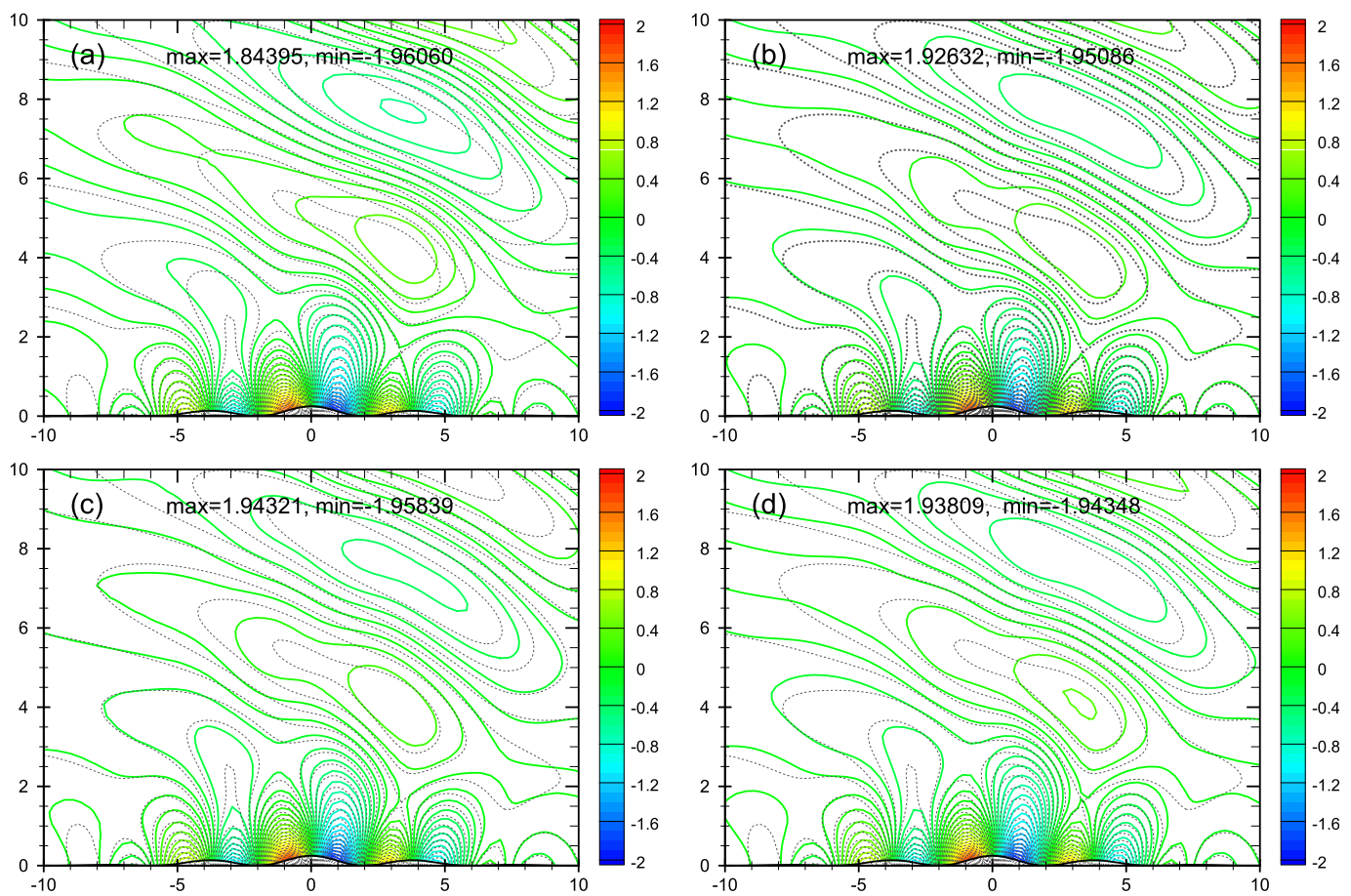

FIG. 10. Vertical velocity perturbation of vertical discretization methods at $10 \mathrm{~h}$ for the test of Schär mountain waves with $\Delta x=0.25 \mathrm{~km}$ and $\Delta z=0.15 \mathrm{~km}$ : (a) 2D SEM, (b) $1 \mathrm{D} \mathrm{VCG,} \mathrm{(c)} \mathrm{1D} \mathrm{VFD,} \mathrm{and} \mathrm{(d)} 1 \mathrm{D}$ VFE. The dotted lines indicate an analytic solution, where its maximum and minimum values are 1.98687 and $-1.98687 \mathrm{~m} \mathrm{~s}^{-1}$, respectively. Spatial units are in kilometers.

coordinates. The first test is presented with no orography and the last three tests simulate mountain waves over single or multiple mountains. In all the tests, the flow patterns obtained from the vertical discretization methods are in good agreement with the analytic and reference solutions as well as the results in the literature. The rising thermal bubble test demonstrates that among the 1D approaches, the VFE method produces more accurate results than the VCG and VFD methods for all grid resolutions. At the medium grid resolution, the error norms evaluated in this test shows that the VFE method achieves much higher accuracy, compared to the VFD method, due to its higher-order spatial accuracy and no orographic effect on the $\sigma$ coordinate. When a small orography is considered, as is the case for the linear hydrostatic or nonhydrostatic mountain waves, the horizontal and vertical velocity perturbations of the 2D SEM are closest to the reference solutions. In the 1D methods, however, the VFE method has the smallest error norms, whereas the VFD provides the worst accuracy. In the Schär mountain wave test, where more complicated and higher mountains are presented, the accuracy trend of the vertical discretizations is consistent with the other mountain tests. The grid dependency study of the vertical discretization methods in all the tests indicates that as the grid resolution increases, the convergence rates of the solutions, expressed in the $L_{2}$ and $L_{\infty}$ error norms, are lower than the level of discretization accuracy, especially in the Schär mountain test, of which the convergence is roughly first order. This slower convergence might be due to the error of a coordinate transformation on orography, which needs to be further investigated in a future study. In terms of efficiency, expressed in wall-clock time, the VCG method

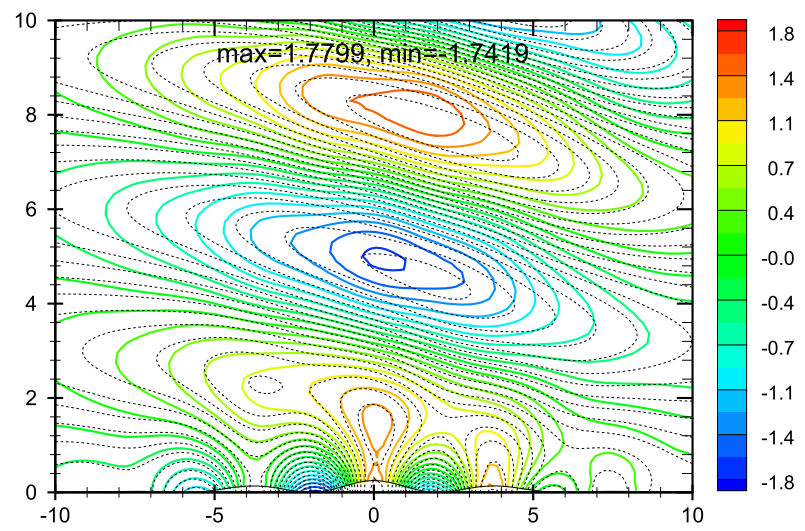

FIG. 11. Horizontal velocity perturbation of the 1D VFE method at $10 \mathrm{~h}$ for the test of Schär mountain waves with $\Delta x=0.25 \mathrm{~km}$ and $\Delta z=0.15 \mathrm{~km}$. The dotted lines indicate an analytic solution, where its maximum and minimum values are 1.7628 and $-2.1183 \mathrm{~m} \mathrm{~s}^{-1}$, respectively. Spatial units are in kilometers. 
TABLE 4. Comparison of the accuracy and efficiency of vertical discretization methods for the test of Schär mountain waves with $\Delta x=$ $0.25 \mathrm{~km}$ and $\Delta z=0.15 \mathrm{~km}$. The fifth-order 2D SEM with $\Delta x=0.11 \mathrm{~km}$ and $\Delta z=0.07 \mathrm{~km}$ is used as a reference solution.

\begin{tabular}{|c|c|c|c|c|c|c|c|}
\hline \multirow[b]{2}{*}{ Methods } & \multicolumn{3}{|c|}{$u^{\prime}\left(\mathrm{m} \mathrm{s}^{-1}\right)$} & \multicolumn{3}{|c|}{$w^{\prime}\left(\mathrm{m} \mathrm{s}^{-1}\right)$} & \multirow[b]{2}{*}{ WT (s) } \\
\hline & $L_{1}$ & $L_{2}$ & $L_{\infty}$ & $L_{1}$ & $L_{2}$ & $L_{\infty}$ & \\
\hline 2D SEM & 0.19261 & 0.19357 & 0.22480 & 0.20488 & 0.18471 & 0.06846 & 14195.2 \\
\hline 1D VCG & 0.26376 & 0.28390 & 0.42137 & 0.26980 & 0.24323 & 0.10179 & 28449.5 \\
\hline 1D VFD & 0.33412 & 0.37096 & 0.55266 & 0.33156 & 0.30384 & 0.13259 & 13220.9 \\
\hline 1D VFE & 0.25399 & 0.27149 & 0.39452 & 0.26411 & 0.23581 & 0.08980 & 22267.9 \\
\hline
\end{tabular}

provides the greatest wall-clock times for all the test cases owing to the inversion of the vertical mass matrix, while the VFD method produces the best efficiency because of its simple discretization method. For the VFE method, expressed in matrix multiplication, the wall-clock time is $148 \%$ greater than that of the 2D SEM when artificial viscosity is required. This is due to the computation of a second derivative operator employed in the VFE method, which is more expensive than that for the VCG and VFD methods. When the second derivative
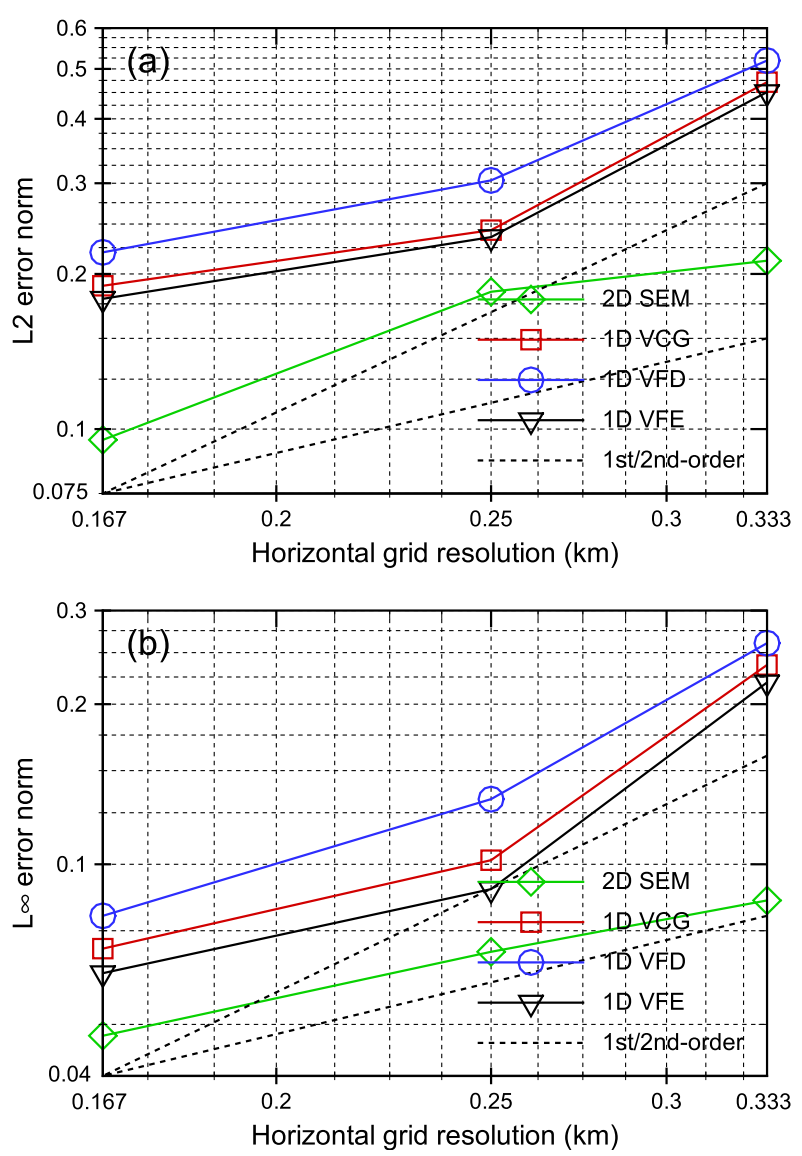

FIG. 12. Grid convergence of vertical discretization methods in the normalized (a) $L_{2}$ and (b) $L_{\infty}$ error norms of vertical velocity perturbation for the test of Schär mountain waves. The axes are in a $\log$ scale. operator is discretized with the central FDM in the framework of the VFE method, the computational time is reduced by $34 \%$ without loss of overall accuracy.

Through all the test cases, the VFE method with the cubic b-spline function on the vertically uniform grid achieves higher accuracy than do the VCG and VFD methods. Moreover, the results show that the VFE method is more efficient than the VCG method, although it is slower than the VFD method, which yields the lowest solution accuracy. This leads to the conclusion that the VFE method is attractive in the vertical discretization when needing to accommodate the physical parameterizations in the high-order spectral element model by considering both accuracy and efficiency. The accuracy of the $1 \mathrm{D}$ decoupling methods might be increased by moving to either a hybrid or more sophisticated vertical coordinate system like SLEVE, in place of the classical Gal-Chen and Somerville $\sigma$ coordinates.

Acknowledgments. This research was supported by the cooperative research and development project with Korea Institute of Atmospheric Prediction Systems under Agreement NCRADA-NPS-13-0181, titled "Development of Nonhydrostatic Dynamical Core on the Sphere: Decoupling Horizontal and Vertical Discretization for the Global Nonhydrostatic System." F. X. Giraldo acknowledges support of the Office of Naval Research through PE-0602435N. Two anonymous reviewers are also gratefully acknowledged for their helpful comments.

\section{REFERENCES}

Abdi, D. S., and F. X. Giraldo, 2016: Efficient construction of unified continuous and discontinuous Galerkin formulations for the 3D Euler equations. J. Comput. Phys., 320, 46-68, https://doi.org/10.1016/j.jcp.2016.05.033.

—- L. C. Wilcox, T. C. Warburton, and F. X. Giraldo, 2019: A GPU-accelerated continuous and discontinuous Galerkin non-hydrostatic atmospheric model. Int. J. High Perform. Comput. Appl., 33, 81-109, https://doi.org/10.1177/ 1094342017694427.

Ahmad, N., and J. Lindeman, 2007: Euler solutions using fluxbased wave decomposition. Int. J. Numer. Methods Fluids, 54, 47-72, https://doi.org/10.1002/fld.1392. 
Appelö, D., and G. Kreiss, 2006: A new absorbing layer for elastic waves. J. Comput. Phys., 215, 642-660, https:// doi.org/10.1016/j.jcp.2005.11.006.

Bao, L., R. Klöfkorn, and R. D. Nair, 2015: Horizontally explicit and vertically implicit (HEVI) time discretization scheme for a discontinuous Galerkin nonhydrostatic model. Mon. Wea. Rev., 143, 972-990, https://doi.org/10.1175/MWR-D14-00083.1.

Blazek, J., 2015: Computational Fluid Mechanics: Principles and Applications. 3rd ed. Elsevier, 466 pp.

Bonaventura, L., 2000: A semi-implicit, semi-Lagrangian scheme using the height coordinate for a nonhydrostatic and fully elastic model of atmospheric flows. J. Comput. Phys., 158, 186-213, https://doi.org/10.1006/jcph.1999.6414.

Dea, J. R., F. X. Giraldo, and B. Neta, 2009: High-order nonreflecting boundary conditions for the linearized 2-D Euler equations: No mean flow case. Wave Motion, 46, 210-220, https://doi.org/10.1016/j.wavemoti.2008.11.002.

Durran, D. R., and J. B. Klemp, 1983: A compressible model for the simulation of moist mountain waves. Mon. Wea. Rev., 111, 2341-2361, https://doi.org/10.1175/1520-0493(1983)111<2341: ACMFTS $>2.0 . C O ; 2$.

ECMWF, 2017: Part III: Dynamics and numerical procedures IFS Documentation-CY43R3, ECMWF, 1-31, https:// www.ecmwf.int/node/17735.

Eskilsson, C., and S. J. Sherwin, 2004: A triangular spectral/hp discontinuous Galerkin method for modelling 2D shallow water equations. Int. J. Numer. Methods Fluids, 45, 605-623, https://doi.org/10.1002/fld.709.

Gal-Chen, T., and R. C. J. Somerville, 1975: On the use of a coordinate transformation for solution of the NavierStokes equations. J. Comput. Phys., 17, 209-228, https:// doi.org/10.1016/0021-9991(75)90037-6.

Gassmann, A., 2005: An improved two-time-level split-explicit integration scheme for non-hydrostatic compressible models. Meteor. Atmos. Phys., 88, 23-38, https://doi.org/10.1007/ s00703-003-0053-8.

Gibbons, S. L., 2009: Impacts of sigma coordinate on the Euler and Navier-Stokes equations using continuous Galerkin methods. M.S. thesis, Naval Postgraduate School, 93 pp., https:// apps.dtic.mil/dtic/tr/fulltext/u2/a496857.pdf.

Giraldo, F. X., 1998: The Lagrange-Galerkin spectral element method on unstructured quadrilateral grids. J. Comput. Phys., 147, 114-146, https://doi.org/10.1006/jcph.1998.6078.

__ , 2006: High-order triangle-based discontinuous Galerkin methods for hyperbolic equations on a rotating sphere. J. Comput. Phys., 214, 447-465, https://doi.org/10.1016/ j.jcp.2005.09.029.

_ , and T. E. Rosmond, 2004: A scalable spectral element Eulerian atmospheric model (SEE-AM) for NWP: Dynamical core tests. Mon. Wea. Rev., 132, 133-153, https://doi.org/ 10.1175/1520-0493(2004)132<0133:ASSEEA > 2.0.CO;2.

_- and M. Restelli, 2008: A study of spectral element and discontinuous Galerkin methods for the Navier-Stokes equations in nonhydrostatic mesoscale atmospheric modeling: Equation sets and test cases. J. Comput. Phys., 227, 3849-3877, https:// doi.org/10.1016/j.jcp.2007.12.009.

__ and _ 2010: High-order semi-implicit time-integrators for a triangular discontinuous Galerkin oceanic shallow water model. Int. J. Numer. Meth., 63, 1077-1102, https:// doi.org/10.1002/fld.2118.

— J. S. Hesthaven, and T. Warburton, 2002: Nodal high-order discontinuous Galerkin methods for the spherical shallow water equations. J. Comput. Phys., 181, 499-525, https:// doi.org/10.1006/jcph.2002.7139.

_ J. F. Kelly, and E. M. Constantinescu, 2013: Implicit-explicit formulations for a three-dimensional nonhydrostatic unified model of the atmosphere (NUMA). SIAM J. Sci. Comput., 35, B1162-B1194, https://doi.org/10.1137/120876034.

Guerra, J. E., and P. A. Ullrich, 2016: A high-order staggered finiteelement vertical discretization for non-hydrostatic atmospheric models. Geosci. Model Dev., 9, 2007-2029, https:// doi.org/10.5194/gmd-9-2007-2016.

Haney, R. L., 1991: On the pressure gradient force over steep topography in sigma coordinate ocean models. J. Phys. Oceanogr., 21, 610-619, https://doi.org/10.1175/1520-0485(1991) 021<0610:OTPGFO $>2.0 . \mathrm{CO} ; 2$.

Kelly, J. F., and F. X. Giraldo, 2012: Continuous and discontinuous Galerkin methods for a scalable three-dimensional nonhydrostatic atmospheric model: Limited-area mode. J. Comput. Phys., 231, 7988-8008, https://doi.org/10.1016/ j.jcp.2012.04.042.

Klemp, J. B., W. C. Skamarock, and O. Fuhrer, 2003: Numerical consistency of metric terms in terrain-following coordinates. Mon. Wea. Rev., 131, 1229-1239, https://doi.org/10.1175/15200493(2003) $131<1229$ :NCOMTI>2.0.CO;2.

Leveque, R. J., 2002: Finite Volume Methods for Hyperbolic Problems. Cambridge University Press, 578 pp.

Li, X., C. Chen, X. Shen, and F. Xiao, 2013: A multimoment constrained finite-volume model for nonhydrostatic atmospheric dynamics. Mon. Wea. Rev., 141, 1216-1240, https://doi.org/ 10.1175/MWR-D-12-00144.1.

Lindquist, J. M., B. Neta, and F. X. Giraldo, 2012: High-order nonreflecting boundary conditions for dispersive waves in polar coordinates using spectral elements. Appl. Math. Comput., 218, 6666-6676, https://doi.org/10.1016/j.amc.2011.12.023.

Lorenz, E. N., 1960: Energy and numerical weather prediction. Tellus, 12, 364-373, https://doi.org/10.3402/tellusa.v12i4.9420.

Marras, S., M. Nazarov, and F. X. Giraldo, 2015: Stabilized highorder Galerkin methods based on a parameter-free dynamic SGS model for LES. J. Comput. Phys., 301, 77-101, https:// doi.org/10.1016/j.jcp.2015.07.034.

Melvin, T., M. Dubal, N. Wood, A. Staniforth, and M. Zerroukat, 2010: An inherently mass-conserving iterative semi-implicit semi-Lagrangian discretization of the non-hydrostatic vertical-slice equations. Quart. J. Roy. Meteor. Soc., 136, 799-814, https://doi.org/10.1002/qj.603.

Müller, A., M. A. Kopera, S. Marras, L. C. Wilcox, T. Isaac, and F. X. Giraldo, 2018: Strong scaling for numerical weather prediction at petascale with the atmospheric model NUMA. Int. J. High Perform. Comput. Appl., 33, 411-426, https:// doi.org/10.1177/1094342018763966.

Nair, R. D., S. J. Thomas, and R. D. Loft, 2005: A discontinuous Galerkin global shallow water model. Mon. Wea. Rev., 133, 876-888, https://doi.org/10.1175/MWR2903.1.

Norman, M., R. Nair, and F. Semazzi, 2011: A low communication and large time step explicit finite-volume solver for nonhydrostatic atmospheric dynamics. J. Comput. Phys., 230, 1567-1584, https://doi.org/10.1016/j.jcp.2010.11.022.

Phillips, N. A., 1957: A coordinate system having some special advantages for numerical forecasting. J. Meteor., 14, 184-185, https://doi.org/10.1175/1520-0469(1957)014<0184: ACSHSS $>2.0 . \mathrm{CO} ; 2$.

Pletcher, R. H., J. C. Tannehill, and D. A. Anderson, 2011: Computational Fluid Mechanics and Heat Transfer. 3rd ed. CRC Press, 774 pp. 
Prenter, P. M., 1975: Splines and Variational Methods. JohnWiley and Sons, $334 \mathrm{pp}$.

Restelli, M., and F. X. Giraldo, 2009: A conservative semi-implicit discontinuous Galerkin method for the Navier-Stokes equations in nonhydrostatic mesoscale atmospheric modeling. SIAM J. Sci. Comput., 31, 2231-2257, https://doi.org/10.1137/ 070708470

Satoh, M., and Coauthors, 2014: The non-hydrostatic icosahedral atmospheric model: Description and development. Prog. Earth Planet. Sci., 1, 1-32, https://doi.org/10.1186/s40645-014-0018-1.

Savre, J., J. Percival, M. Herzog, and C. Pain, 2016: Two-dimensional evaluation of ATHAM-Fluidity, a nonhydrostatic atmospheric model using mixed continuous/discontinuous finite elements and anisotropic grid optimization. Mon. Wea. Rev., 144, 4349 4372, https://doi.org/10.1175/MWR-D-15-0398.1.

Schär, C., D. Leuenberger, O. Fuhrer, D. Lüthi, and C. Girard, 2002: A new terrain-following vertical coordinate formulation for atmospheric prediction models. Mon. Wea. Rev., 130, 2459-2480, https://doi.org/10.1175/1520-0493(2002)130<2459: ANTFVC $>2.0 . \mathrm{CO} ; 2$.

Shu, C.-W., and S. Osher, 1988: Efficient implementation of essentially non-oscillatory shock-capturing schemes. J. Comput. Phys., 77, 439-471, https://doi.org/10.1016/0021-9991(88)90177-5.

Simarro, J., and M. Hortal, 2012: A semi-implicit non-hydrostatic dynamical kernel using finite elements in the vertical discretization. Quart. J. Roy. Meteor. Soc., 138, 826-839, https:// doi.org/10.1002/qj.952.

Simmons, A. J., and D. M. Burridge, 1981: An energy and angularmomentum conserving vertical finite-difference scheme and hybrid vertical coordinates. Mon. Wea. Rev., 109, 758-766, https://doi.org/10.1175/1520-0493(1981)109<0758:AEAAMC> 2.0. $\mathrm{CO} ; 2$

Skamarock, W. C., and J. B. Klemp, 2008: A time-split nonhydrostatic atmospheric model for weather research and forecasting applications. J. Comput. Phys., 227, 3465-3485, https://doi.org/10.1016/j.jcp.2007.01.037.

Spiteri, R. J., and S. J. Ruuth, 2002: A new class of optimal highorder strong-stability-preserving time discretization methods. SIAM J. Numer. Anal., 40, 469-491, https://doi.org/10.1137/ S0036142901389025.

Staniforth, A. N., and R. W. Daley, 1977: A finite-element formulation for the vertical discretization of sigma coordinate primitive equation models. Mon. Wea. Rev., 105, 1108-1118, https://doi.org/ 10.1175/1520-0493(1977)105<1108:AFEFFT>2.0.CO;2.

Sundqvist, H., 1975: On truncation errors in sigma-system models. Atmosphere, 13, 81-95, https://doi.org/10.1080/ 00046973.1975 .9648390 .

Taylor, M. A., and A. Fournier, 2010: A compatible and conservative spectral element method on unstructured grids. J. Comput. Phys., 229, 5879-5895, https://doi.org/10.1016/ j.jcp.2010.04.008.

Thomas, S. J., and R. Loft, 2005: The NCAR spectral element climate dynamical core: Semi-implicit Eulerian formulation. J. Sci. Comput., 25, 307-322, https://doi.org/10.1007/s10915004-4646-2.

Thuburn, J., 2011: Vertical discretizations: Some basic ideas. Numerical Techniques for Global Atmospheric Models, P. H. Lauritzen et al., Eds., Springer, 59-74.

Untch, A., and M. Hortal, 2004: A finite-element scheme for the vertical discretization of the semi-Lagrangian version of the ECMWF forecast model. Quart. J. Roy. Meteor. Soc., 130, 1505-1530, https://doi.org/10.1256/qi.03.173.

Vivoda, J., P. Smolíková, and J. Simarro, 2018: Finite elements used in the vertical discretization of the fully compressible core of the ALADIN system. Mon. Wea. Rev., 146, 3293-3310, https://doi.org/10.1175/MWR-D-18-0043.1.

Walters, D. N., and Coauthors, 2011: The Met Office Unified Model Global Atmosphere 3.0/3.1 and JULES Global Land 3.0/3.1 configurations. Geosci. Model Dev., 4, 919-941, https:// doi.org/10.5194/gmd-4-919-2011.

Weller, H., and A. Shahrokhi, 2014: Curl-free pressure gradients over orography in a solution of the fully compressible Euler equations with implicit treatment of acoustic and gravity waves. Mon. Wea. Rev., 142, 4439-4457, https://doi.org/ 10.1175/MWR-D-14-00054.1

Xue, M., K. K. Droegemeier, and V. Wong, 2000: The Advanced Regional Prediction System (ARPS) - A multi-scale nonhydrostatic atmospheric simulation and prediction model. Part I: Model dynamics and verification. Meteor. Atmos. Phys., 75, 161-193, https://doi.org/10.1007/s007030070003.

Yi, T.-H., and J.-R. Park, 2017: Vertical discretization with finite elements for a global hydrostatic model on the cubed sphere. J. Comput. Phys., 338, 339-356, https://doi.org/10.1016/ j.jcp.2017.02.067. 